\title{
Capacity of Hybrid Cognitive Radio Networks With Distributed VAAs
}

\author{
Xuemin Hong, Cheng-Xiang Wang, Senior Member, IEEE, Murat Uysal, Senior Member, IEEE, \\ Xiaohu Ge, Member, IEEE, and Shan Ouyang
}

\begin{abstract}
A cooperative hybrid cognitive radio (CR) network is proposed to simultaneously operate on a dedicated licensed band and a secondary band. The licensed band is used for communications between a base station (BS) and mobile CR users, whereas the secondary band is used to facilitate the licensed band communication by coordinating multiple CR users to form distributed virtual antenna arrays (VAAs). The capacity of the proposed CR network is studied at both the link and system levels. At the link level (single VAA case), we present an amplify-andforward-based cooperative signaling scheme that employs power control to prevent harmful noise propagation. The resulting virtual multiple-input-multiple-output (MIMO) link capacity is derived and compared with the real MIMO system. At the system level (multiple VAAs case), the system capacity is derived as a function of multiple parameters, including the primary user density, CR user density, primary exclusion region radius, and VAA radius. Under an average interference power constraint, the maximum system capacity is further calculated by solving an optimization problem with adjustable system parameters. $\mathrm{Nu}$ -
\end{abstract}

Manuscript received November 3, 2009; revised February 27, 2010 and May 25, 2010; accepted May 31, 2010. Date of publication June 14, 2010; date of current version September 17, 2010. The work of X. Hong and C.-X. Wang was supported by the Scottish Funding Council for the Joint Research Institute in Signal and Image Processing with the University of Edinburgh, as part of the Edinburgh Research Partnership in Engineering and Mathematics (ERPem). The work of X. Hong, C.-X. Wang, and X. Ge (Corresponding author) was supported by the RCUK for the U.K.-China Science Bridges: R\&D on (B) 4G Wireless Mobile Communications. The work of M. Uysal was supported in part by the Natural Sciences and Engineering Research Council of Canada under Discovery Grants Accelerator Supplement 363168-2008. The work of X. Ge was supported in part by the National Natural Science Foundation of China (NSFC) under Grant 60872007, by the National 863 High Technology Program of China under Grant 2009AA01Z239, and by the Ministry of Science and Technology (MOST) of China, International Science and Technology Collaboration Program, under Grant 0903. The work of S. Ouyang was supported in part by the National Basic Research Program of China "973" under Grant 2008CB317109, by Guangxi Science Foundation under Grant 0991241 , by NSFC under Grants 60872022 and 60972084, and by the Foundation of Guangxi Key Laboratory of Information and Communication under Grant 10903. The work of C.-X. Wang and S. Ouyang was supported by the Key Laboratory of Cognitive Radio and Information Processing (Guilin University of Electronic Technology), Ministry of Education. The review of this paper was coordinated by Prof. B. Hamdaoui.

X. Hong and C.-X. Wang are with the Joint Research Institute for Signal and Image Processing, School of Engineering and Physical Sciences, HeriotWatt University, Edinburgh EH14 4AS, U.K. (e-mail: x.hong@hw.ac.uk; cheng-xiang.wang@hw.ac.uk).

M. Uysal is with the Faculty of Engineering, Özyeğin University, 34662 Istanbul, Turkey (e-mail: murat.uysal@ozyegin.edu.tr).

$\mathrm{X}$. Ge is with the Department of Electronics and Information Engineering, Huazhong University of Science and Technology, Wuhan 430074, China (e-mail: xhge@mail.hust.edu.cn).

S. Ouyang is with the Department of Communications and Information Engineering, Guilin University of Electronic Technology, Guilin 541004, China (e-mail: hmoysh@guet.edu.cn).

Color versions of one or more of the figures in this paper are available online at http://ieeexplore.iee.org.

Digital Object Identifier 10.1109/TVT.2010.2053056 merical studies reveal that the proposed cooperative hybrid CR network has a fundamental advantage over a pure CR network by being insensitive to the characteristics of the coexisting primary network. This merit, however, relies on a high CR user density and a wide bandwidth of the secondary band.

Index Terms-Capacity analysis, cognitive radio (CR) network, multiple-input multiple-output (MIMO), virtual antenna array (VAA).

\section{INTRODUCTION}

C URRENT spectrum-regulation policies have resulted in an imbalanced and inefficient utilization of the precious radio spectrum. Certain bands are highly congested, whereas a large portion of the spectrum is only sparsely used across geographical areas and time [1]. Over recent years, cognitive radio (CR) [2]-[6] has been pursued as a promising technology to improve the spectrum utilization by enabling secondary usage of the underutilized spectrum licensed to primary systems. A secondary CR network is able to adapt to dynamic spectrum environments and achieve its communication goals without jeopardizing cochannel primary services.

CR networks can broadly be categorized into two types, namely, interweave CR networks and overlay CR networks [5]. The former are also called noninterfering CR networks [6]. They detect spectrum holes via sensing [3] and adaptively tune secondary radio transceivers to operate in the orthogonal signal space of primary transmissions. The main research concern is how to reliably discover spectrum holes scattered in a wide frequency range and effectively use them. The interweave CR network offers a practical solution to achieve coexistence of primary and secondary networks. It has been the main focus of $\mathrm{CR}$ research and has drawn standardization efforts from the IEEE 802.22 [7] working group.

Overlay CR networks are also called interference-tolerant CR networks [6]. They take a more fundamental view on coexistence by shifting the focus from primary transmitters to primary receivers. Secondary transmissions are allowed as long as the actual interference perceived at the primary receivers is controlled to fulfill certain protective constraints. The unique research problem is how to reliably manage the interference at primary receivers. In practice, certain feedback mechanisms are required to periodically inform CR transmitters about the instantaneous interference level at the primary receivers. For instance, primary receivers can either report the measured interference levels via a dedicated common control channel [8] or transmit pilot signals, which can be sensed by CR transmitters to estimate the interference channel gain [9]. Due to the need of primary receiver feedback, overlay CR 
networks are not suitable to be deployed in certain bands, e.g., TV bands, but are more appropriate in bands with relatively sparse primary receivers (e.g., radar or fixed microwave communication (FMC) bands). Despite facing greater technical and regulatory challenges, overlay $\mathrm{CR}$ networks can achieve better spectrum utilization than interweave CR networks by allowing secondary networks to overlap with primary networks in the same signal space. This advantage can make the overlay $\mathrm{CR}$ network a favorable solution in certain scenarios where spectrum holes are limited, fragmented, or unusable (e.g., the example in Section IV). Unlike interweave CR networks that are becoming a commercial reality, overlay CR networks still face open questions with regard to their fundamental limits, practicality, and design guidelines. In this paper, we focus on overlay CR networks, which will be simply referred to as $\mathrm{CR}$ networks subsequently.

Pure CR networks utilizing only the secondary spectrum (i.e., licensed spectrum opened for secondary use) have unreliable performance because the availability of the secondary spectrum is susceptible to the behavior of the coexisting primary networks. A number of studies [10]-[13] have shown that the capacities of pure CR networks can greatly fluctuate even with slight changes in the densities, interference tolerances, or transmission behaviors of the primary users. This instability of the capacity motivated us to propose the hybrid $C R$ network, which combines an opportunistic pure CR network with a reliable licensed network and seeks to leverage the advantages of both networks [6]. For example, a cellular network can explore secondary bands outside its licensed cellular band and form a hybrid CR network to expand its capacity.

Two architectures exist for hybrid CR networks, namely, noncooperative and cooperative [6]. A noncooperative CR network has two independent radio physical layers (PHYs) separately operating in the licensed and secondary bands. CR devices can adaptively select a PHY interface that best suits their communication tasks. For example, services with strict qualityof-service (QoS) requirements can be scheduled to the (reliable) licensed PHY, whereas services with relaxed QoS requirements can be delivered over the (opportunistic) secondary PHY. The system capacity of a noncooperative hybrid CR network is simply the sum of the capacities provided by the licensed and secondary PHYs. In many secondary bands, tight transmit power constraints are imposed to protect primary systems. Consequently, the capacity of the secondary network usually quickly diminishes with increasing communication ranges [11], making a noncooperative hybrid CR network only worthwhile to deploy for short- to medium-range communications.

Alternatively, cooperative hybrid CR networks were proposed in [6] to have a single integrated PHY across both bands: the licensed band is used to send information to the (distant) destination, whereas the secondary band is used to facilitate the licensed band transmission using certain forms of cooperative communications [14]. For example, a CR source can use the secondary band to coordinate nearby CR users to form a virtual antenna array (VAA) [15]. The VAA can then bring significant capacity gains over the licensed band by emulating multiple-input-multiple-output (MIMO) communications [16]. Compared with noncooperative systems, coop- erative hybrid CR networks have major advantages in being able to support medium- to long-range communications and providing better immunity against high primary user density [6]. Intuitively, these advantages come from exploiting the typically complementary characteristics of the licensed and secondary radio resources. The licensed spectrum, which has a relatively small bandwidth but can operate at high power levels, is better used for long-range communications. On the other hand, the secondary spectrum, which has tight power limits but a potentially wide bandwidth, is more suitable for short-range communications to facilitate local cooperation.

The cooperative hybrid CR network studied in [6] was largely oversimplified. In this paper, we aim to propose and study a novel cooperative hybrid CR network, which is more realistic and advanced in three aspects.

1) A time-sharing system was assumed in [6], allowing one VAA to be active at a time (in a cell). Contrary to [6], this paper considers a distributed VAA system that allows multiple VAAs to simultaneously operate to bring significant increases to the system capacity.

2) A frequency-division multiple-access (FDMA) scheme was assumed in [6] for transmissions in the secondary band. This scheme is replaced in this paper by codedivision multiple access (CDMA). This change is nontrivial since CDMA provides an inherent advantage to exploit the wideband feature of the secondary band to improve the system performance.

3) Similar to [6], we assume amplify-and-forward relaying in this paper. This scheme is preferable for delay-constrained services but requires high signal-tointerference-and-noise ratio (SINR) in the source-to-relay link to avoid harmful noise propagation. While there is no mechanism provided in [6] to control noise propagation, a refined VAA signaling scheme with power control is proposed in this paper to prevent harmful noise propagation.

The capacity of the proposed cooperative hybrid CR network will be studied first at the link level and subsequently at the system level. We study the uplink capacity when only one secondary band is used, noting that extensions to the downlink capacity and multiple secondary bands are straightforward. These capacity studies serve to measure the long-term performance limits of large-scale cooperative hybrid CR networks and provide guidelines to strategic network planning. We show that the proposed cooperative hybrid CR network is a promising alternative to the noncooperative hybrid $\mathrm{CR}$ networks to be deployed in urban scenarios to provide reliable medium- to long-range communication services.

The remainder of this paper is organized as follows: Section II describes the link model of VAA signaling and analyzes the resulting link capacity. The system capacity involving multiple VAA links is subsequently derived in Section III. Numerical results and discussion are provided in Section IV. Finally, conclusions are drawn in Section V.

\section{LINK-LEVEL CAPACITY ANALYSIS}

We consider a centralized hybrid cooperative CR network where a base station (BS) with $M_{r}$ antenna elements located 


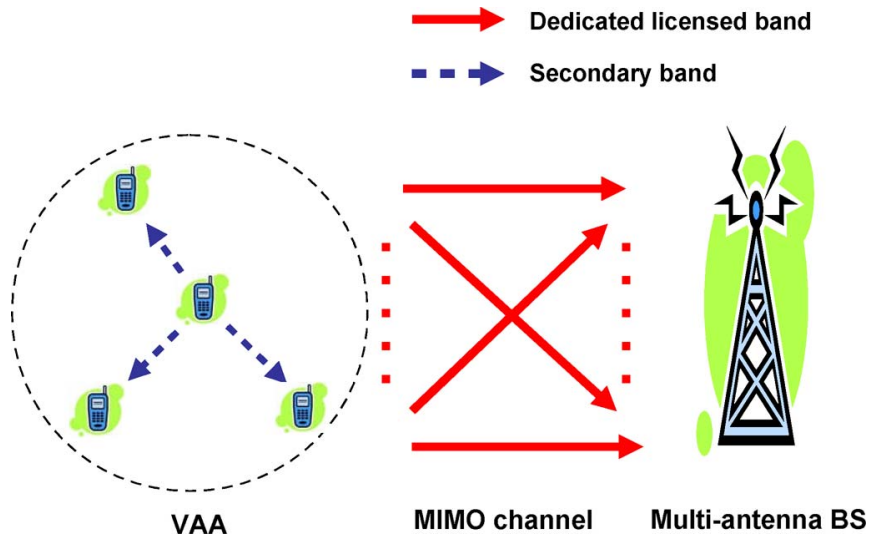

Fig. 1. Link model of a cooperative hybrid CR network with a VAA and a multiantenna BS.

at a cell center communicates with multiple single-antenna $\mathrm{CR}$ users distributed in the cell. The network can operate on a dedicated licensed band and a secondary band. The licensed band is exclusively used for communications between the BS and CR users, whereas the secondary bands are exclusively used for neighboring $\mathrm{CR}$ users to self-organize into multiple ad hoc VAA groups.

This section focuses on the link-level communication, which involves only one VAA and the BS. Fig. 1 illustrates the link model and shows two phases in a virtual MIMO communication signaling scheme. In the first phase, the target CR user sends relevant information to cooperating users in the VAA. Communications in this phase is performed in the secondary band with best effort, under an interference constraint that protects the cochannel primary network from the CR interference. In the second phase, the VAA acts as a whole to communicate with the BS in the dedicated licensed band. Four basic assumptions are made in our following discussions: First, to protect the primary network, a CR VAA has a maximum total power $P$ to transmit in the secondary band. Second, an amplify-and-forward scheme is used in the relaying process. Third, CDMA is used for all transmissions in the secondary band. Fourth, the CR users can simultaneously and independently operate in the dedicated licensed band and the secondary band. This assumption is feasible if these two bands are sufficiently separated.

\section{A. VAA Establishment}

The first task of a target CR user is to coordinate neighboring idle CR users to establish a VAA. We propose the following protocol to achieve this with two handshakes and four steps.

1) The target $C R$ user broadcasts relevant information with full power $P$ in a preselected broadcast channel.

2) Idle CR users periodically monitor the broadcast channel and evaluate the SINR of the received signals. Once the SINR is detected to be above a certain threshold, the idle CRs send feedback information (including, e.g., user identification and SINR information) to the target CR through slotted random access channels with power $P$.

3) Based on the feedback information from idle CR users, the target user selects those with good channel qualities to form a VAA. The selection criterion is that those selected
CR users can simultaneously receive independent signals from the target user, through orthogonal CDMA channels and with a minimum SINR $\rho_{\text {cr }}$. This selection criterion guarantees that only signals with high qualities are forwarded in the second phase and thereby prevents harmful noise propagation [17]. It can be noted that classic cellular CDMA downlink power control algorithms such as that proposed in [18] can be used to select CR users into a VAA. Once the selection is completed, the target CR user will broadcast the identities of selected idle users and allocate a channel [Pseudo-Noise (PN) code] to each one.

4) Finally, the selected idle users acknowledge their cooperation and tune to the allocated CDMA channels to receive data. After a VAA is formed, we will subsequently call the selected idle users as cooperating users. The number of single-antenna users (including the target user) in a VAA is denoted by $M_{t}$.

\section{B. Transmissions in the Secondary Band}

After a VAA is established (with $M_{t}$ transmit antennas), the actual virtual MIMO communication can start. Suppose that the target user has an information vector, which is denoted $\mathbf{s}=\left[s_{1}, \ldots, s_{M_{t}}\right]^{T}$, intended to be transmitted at certain time slot from the VAA to the destination. Here, each symbol within the vector $\mathbf{s}$ has unit power, and $[\cdot]^{T}$ represents the transpose of a matrix. Since the target user with a single antenna can only transmit one symbol $s_{M_{t}}$ at a time slot, it should transmit other information symbols $s_{q}\left(q=1, \ldots, M_{t}-1\right)$ to the corresponding $M_{t}-1$ cooperating users in the previous time slot via orthogonal CDMA channels in the secondary band. These channels between the target user and cooperating users are called cooperation channels. Realistic modeling of such channels can be found in [19]-[21]. We assume that a power control scheme, such as that in [18], is employed by the target $\mathrm{CR}$ user to compensate the channel loss in the cooperation channels so that the received symbols (after CDMA despreading) at all the cooperating users have the same SINR $\rho_{\text {cr }}$. With an amplify-and-forward relaying scheme, the symbols received by cooperative users at a previous time slot are amplified and retransmitted in the licensed band in the next time slot. It follows that the retransmitted symbols (with power normalized to 1 ) from the $q$ th cooperation user is given by

$$
\hat{s}_{q}=\sqrt{\frac{1+\rho_{\mathrm{cr}}}{\rho_{\mathrm{cr}}}}\left(\exp \left(i \theta_{q}\right) s_{q}+\sqrt{\frac{1}{\rho_{\mathrm{cr}}}} \hat{n}_{q}\right)
$$

where $i=\sqrt{-1}, \exp \left(i \theta_{q}\right)$ represents the random phase shift in the $q$ th cooperation channel, and $\hat{n}_{q}$ corresponds to the interference-plus-noise term at the $q$ th cooperating user. We assume that $\hat{n}_{q}$ can be treated as additive white Gaussian noise (AWGN) with unit power. The signal vector actually transmitted from the VAA is then given by $\hat{\mathbf{s}}=\left[\hat{s}_{1}, \ldots, \hat{s}_{M_{t}-1}, s_{M_{t}}\right]^{T}$, and we have

$$
\hat{\mathbf{s}}=\sqrt{\frac{\rho_{\mathrm{cr}}}{1+\rho_{\mathrm{cr}}}} \hat{\mathbf{H}} \mathbf{s}+\sqrt{\frac{1}{1+\rho_{\mathrm{cr}}}} \hat{\mathbf{n}}
$$


where $\hat{\mathbf{H}}$ is the $M_{t} \times M_{t}$ cooperation channel-phase matrix given by

$$
\hat{\mathbf{H}}=\operatorname{diag}\left(\exp \left(i \theta_{1}\right), \exp \left(i \theta_{2}\right), \ldots, \exp \left(i \theta_{M_{t}-1}\right), 1\right)
$$

and $\hat{\mathbf{n}}$ is the $M_{t} \times 1$ noise vector given by

$$
\hat{\mathbf{n}}=\left[\hat{n}_{1}, \hat{n}_{2}, \ldots, \hat{n}_{M_{t}-1}, 0\right]^{T} .
$$

\section{Transmissions in the Licensed Band}

The VAA with $M_{t}$ transmit antennas and the BS antenna array with $M_{r}$ receive antennas form an $M_{t} \times M_{r}$ virtual MIMO communication link in the dedicated licensed band. Let us denote the $M_{t} \times M_{r}$ MIMO channel-transfer matrix in the licensed band by $\tilde{\mathbf{H}}$. When an $M_{t} \times 1$ signal vector $\hat{\mathbf{s}}$ given by (2) is transmitted from the VAA, the $M_{r} \times 1$ received signal vector $\mathbf{y}$ at the BS is given by [16]

$$
\mathbf{y}=\sqrt{\frac{\rho_{\mathrm{bs}}}{M_{t}}} \tilde{\mathbf{H}} \hat{\mathbf{s}}+\tilde{\mathbf{n}}
$$

where $\rho_{\text {bs }}$ denotes the average SINR at the BS, and $\tilde{\mathbf{n}}$ is an $M_{r} \times 1$ vector whose elements represent the interference-plusnoise signal at the BS antenna elements. Each element of $\tilde{\mathbf{n}}$ is assumed to be AWGN with unit power. Substituting (2) into (5), we get

$$
\mathbf{y}=\sqrt{\frac{\rho_{\mathrm{bs}}}{M_{t}}} \sqrt{\frac{\rho_{\mathrm{cr}}}{1+\rho_{\mathrm{cr}}}} \mathbf{H s}+\mathbf{n}
$$

where $\mathbf{H}$ is the virtual MIMO channel matrix given by the product of the cooperation channel-phase matrix $\hat{\mathbf{H}}$ and the licensed band MIMO channel matrix $\tilde{\mathbf{H}}$, i.e.,

$$
\mathbf{H}=\tilde{\mathbf{H}} \hat{\mathbf{H}} \text {. }
$$

In (6), $\mathbf{n}$ is defined as

$$
\mathbf{n}=\sqrt{\frac{\rho_{\mathrm{bs}}}{M_{t}}} \sqrt{\frac{1}{1+\rho_{\mathrm{cr}}}} \tilde{\mathbf{H}} \hat{\mathbf{n}}+\tilde{\mathbf{n}}
$$

which represents the total interference-plus-noise signal. It includes not only the local interference-plus-noise signal at the BS but the regenerative interference-plus-noise signal forwarded from the VAA as well.

\section{Link Capacity}

Given (6), the link capacity of the virtual MIMO channel is defined as the maximum mutual information between the vectors $\mathbf{y}$ and s. Following similar steps in [16], the normalized ergodic link capacity (spectral efficiency) of the virtual MIMO channel in the absence of channel knowledge at the transmitter can easily be derived as

$$
C_{L}\left(M_{t}\right)=\mathbb{E}\left\{\log _{2}\left[\operatorname{det}\left(\mathbf{I}_{M_{r}}+\frac{\rho_{\mathrm{bs}}}{M_{t}} \frac{\rho_{\mathrm{cr}}}{1+\rho_{\mathrm{cr}}} \mathbf{R}_{n n}^{-1} \mathbf{H H}^{\dagger}\right)\right]\right\}
$$

where $\mathbb{E}(\cdot)$ is the expectation operator, $(\cdot)^{\dagger}$ denotes the Hermitian transpose of a matrix, $\mathbf{I}_{M_{r}}$ is an $M_{r} \times M_{r}$ identity matrix, and $\mathbf{R}_{n n}=\mathbb{E}\left\{\mathbf{n} \mathbf{n}^{\dagger}\right\}$ is the $M_{r} \times M_{r}$ covariance matrix of the noise vector $\mathbf{n}$. From (8), we can calculate $\mathbf{R}_{n n}$ as

$$
\mathbf{R}_{n n}=\mathbf{I}_{M_{r}}+\frac{\rho_{\mathrm{bs}}}{M_{t}} \frac{1}{1+\rho_{\mathrm{cr}}} \tilde{\mathbf{H}} \mathbf{R}_{\hat{n} \hat{n}} \tilde{\mathbf{H}}^{\dagger}
$$

where $\mathbf{R}_{\hat{n} \hat{n}}$ is the $M_{t} \times M_{t}$ covariance matrix of the vector $\hat{n}$. From (4), we have

$$
\mathbf{R}_{\hat{n} \hat{n}}=\operatorname{diag}[1,1, \ldots, 1,0] .
$$

The expectation in (9) is taken over the fading states of the virtual MIMO channel, i.e., the random matrix $\mathbf{H}$ given by (7). From (9), it is obvious that given certain channel-fading statistics, the normalized ergodic link capacity $\rho_{\text {cr }}$ is determined by three parameters: the average SINR at cooperating CR users, the average SINR $\rho_{\mathrm{bs}}$ at the BS, and the number of transmit/receive antenna elements. The impacts of these three parameters on the ergodic capacity will subsequently be investigated using numerical methods. The channel coefficients in $\tilde{\mathbf{H}}$ are assumed to be independent and are obtained from a composite channel model, including lognormal shadowing (with a standard deviation of $8 \mathrm{~dB}$ ) and Rayleigh fading (with unit power). We assume $M_{r}=8$ as an example for a highperformance BS. For comparison purposes, here, we also give the classic normalized ergodic link capacity of a real $M_{t} \times M_{r}$ MIMO system [16], i.e.,

$$
\tilde{C}_{L}\left(M_{t}\right)=\mathbb{E}\left\{\log _{2}\left[\operatorname{det}\left(\mathbf{I}_{M_{r}}+\frac{\rho_{\mathrm{bs}}}{M_{t}} \tilde{\mathbf{H}} \tilde{\mathbf{H}}^{\dagger}\right)\right]\right\}
$$

where the expectation is taken over the random channel matrix $\tilde{\mathbf{H}}$. It is obvious that (9) differs from (12) by taking into account extra factors such as the CR channel SNR $\rho_{\text {cr }}$ and the CR VAA channel matrix $\hat{\mathbf{H}}$.

Fig. 2 shows the normalized ergodic link capacity of a virtual MIMO link $C_{L}$ given by (9) as a function of $\rho_{\mathrm{bs}}$ with different values of $\rho_{\text {cr }}$ and $M_{t}$. The normalized ergodic link capacities $\tilde{C}_{L}$ of real MIMO systems given by (12) are also shown for comparisons. Clearly, with the increase of $M_{t}$, both the real and virtual MIMO channel capacities increase. Moreover, large $\rho_{\text {cr }}$ makes the resulting ergodic virtual MIMO channel capacity approach closely to the corresponding real MIMO channel capacity. When $M_{t}=1$, both the real MIMO and virtual MIMO systems reduce to a single-input-singleoutput system. In Fig. 3, we show the normalized ergodic link capacity of virtual MIMO as a function of $\rho_{\mathrm{cr}}$ with $\rho_{\mathrm{bs}}=$ $8 \mathrm{~dB}$, which is a typical value in, e.g., cellular communication systems. It is observed that, for the same number of transmit antennas, virtual MIMO gives a lower normalized ergodic link capacity than real MIMO schemes due to the noise caused in the relay (i.e., amplify-and-forward) process. Higher values of $\rho_{\text {cr }}$ mean smaller noise in the relay process, and hence, the normalized ergodic link capacity of virtual MIMO approaches that of real MIMO when $\rho_{\text {cr }}$ is sufficiently high. A $\rho_{\text {cr }}$ value of $15 \mathrm{~dB}$ is required to achieve about $80 \%$ of the real MIMO capacity gain. 


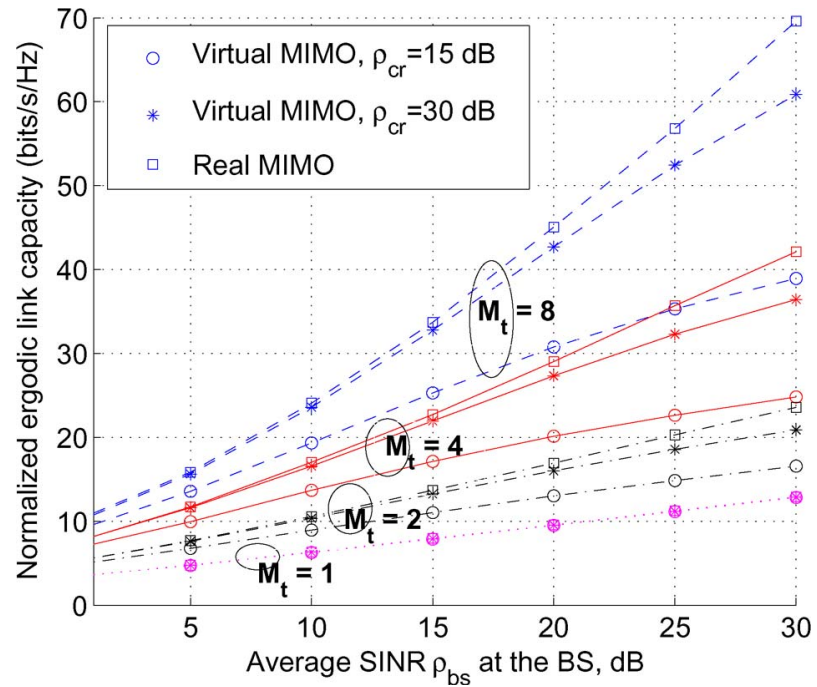

Fig. 2. Normalized ergodic link capacities of a virtual MIMO channel $C_{L}$ and a real MIMO channel $\tilde{C}_{L}$ as functions of the average received SINR $\rho_{\mathrm{bs}}$ at the BS with different VAA transmit antenna numbers $M_{t}$ and $\rho_{\text {cr }}\left(M_{r}=8\right)$.

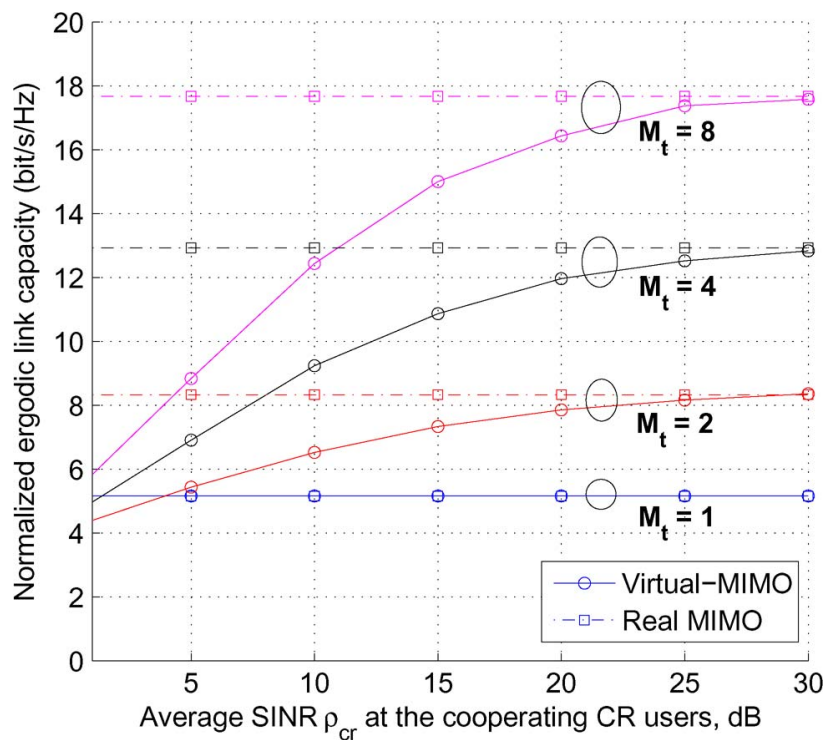

Fig. 3. Normalized ergodic link capacities of a virtual MIMO channel $C_{L}$ and a real MIMO channel $\tilde{C}_{L}$ as functions of the average received SINR $\rho_{\text {cr }}$ at the CR relays with different VAA transmit antenna numbers $M_{t}\left(\rho_{\mathrm{bs}}=\right.$ $8 \mathrm{~dB}$, and $\left.M_{r}=8\right)$.

\section{System-LEVEl CAPACITY ANALYSIS}

\section{A. System Model}

In this section, we will study the capacity of the proposed hybrid CR network at the system level. Fig. 4 illustrates the system model consisting of multiple primary users, CR users, and a BS. The primary users/receivers (illustrated as disk antennas) and CR users (illustrated with mobile phones) are randomly distributed on a plane. We assume that the spatial distributions of the primary and CR users can be described by two stationary Poisson point processes with density parameters $\lambda_{p}$ and $\lambda_{c}$, respectively. These density parameters denote the average number of users per unit area. The spatial Poisson model is adopted because it is a powerful analytical tool to

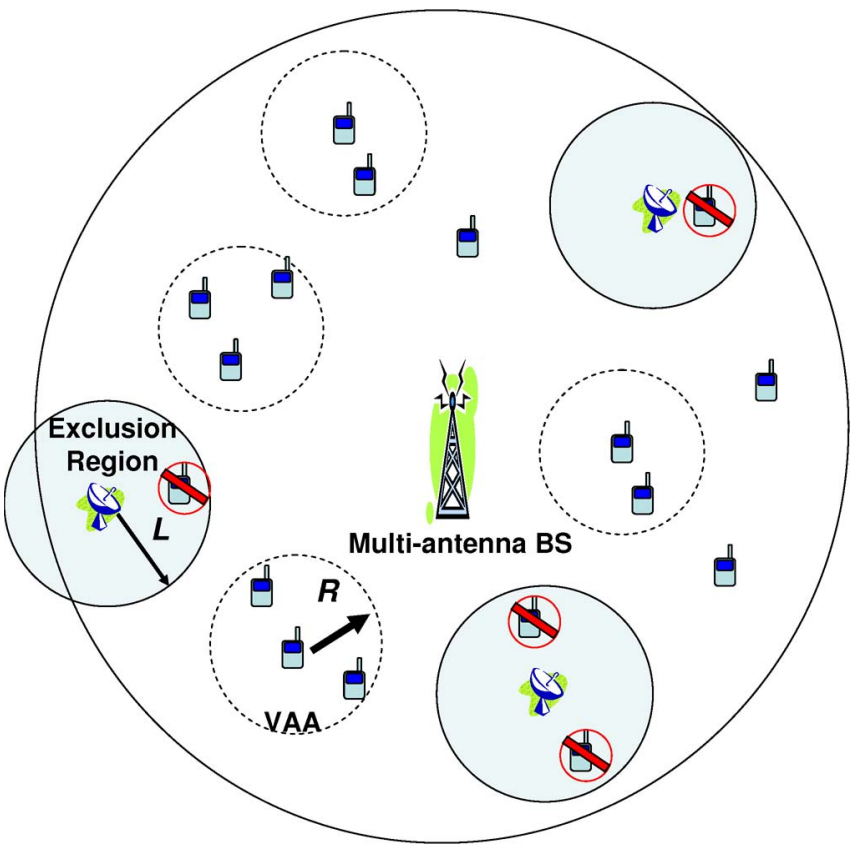

Fig. 4. System model of a cooperative hybrid CR network with a BS, multiple primary users, and multiple CR users.

describe the random spatial distributions of mobile users and has intensively been used in system-level performance studies of mobile networks (e.g., [22] and [23]).

Centered at each primary receiver, there is an "exclusion region" [5], [24], [25]. Any CR user within this exclusion region is forbidden to transmit in the secondary band. Previous studies [5], [24], [25] have shown that the exclusion region is an effective mechanism to protect primary receivers by eliminating dominant interferers and reduce the aggregate interference. To detect exclusion regions in practice, a CR can either detect the special beacons [8] transmitted by primary receivers, sense the RF leakage emitted by primary receivers [9], or consult a primary receiver location database [7]. These schemes will lead to exclusion regions of circular shapes under the assumption of path-loss-only (i.e., nonfading) channel models, which are widely used for system-level capacity analysis [6], [10], [22][24]. We assume that all primary receivers are equally protected with the same exclusion region radius $L$. It is worth noting that it is more appropriate to implement "exclusion region" in primary networks with relatively sparse receivers (e.g., radar and FMC networks) so that the implementation cost is relatively low and the remained regions allowing CR transmissions are large enough to justify the deployment of CR networks.

Outside the exclusion regions, CR users can communicate in the secondary band to form multiple VAAs. These VAAs are established in a distributed and ad hoc fashion to simultaneously and opportunistically improve the communications of multiple target users. To increase the capacity of a cooperative hybrid CR network, a secondary band should spatially be reused to simultaneously establish many distributed VAAs without interfering with each other. To mitigate the mutual interference among VAAs, we assume that every VAA has a circular region of radius $R$ and different VAAs cannot overlap. We denote the spatial densities of the VAAs and CR users transmitting 
in the licensed band by $\lambda_{v}$ and $\lambda_{l}$, respectively. Clearly, we have $\lambda_{v} \leq \lambda_{l} \leq \lambda_{c}$. When VAAs are established in multiple secondary bands, the total VAA spatial density is simply the sum of the densities in each band.

\section{B. Interference Modeling and Approximation}

Due to their spectrum-sharing nature, $\mathrm{CR}$ networks inevitably operate in interference-intensive environments. Before we can analyze the system capacity of the proposed hybrid CR network, we need to first understand the characteristics of the interference in the secondary band. Three types of interference in the secondary band are of our interest. The first type is the aggregate interference $I_{p}$ received at a primary receiver from distributed VAAs. This interference is of great importance in CR networks since it should be bounded by certain constraints to protect the primary services. The second type is the aggregate interference $I_{s}$ received at a VAA from other VAAs. This mutual interference among VAAs could become a limiting factor in a multiuser context and should properly be treated in system-level studies. The third type is the interference from the primary network to VAAs. Usually, this interference does not significantly vary over time and space and can be treated as the background interference with a constant power. We will subsequently use $I_{0}$ to denote the background interferenceplus-noise power perceived by the secondary receivers.

Let us first study the aggregate interference $I_{p}$ at a primary receiver. After a VAA is established, only the target user transmits in the secondary band. Therefore, the entire distributed VAA network has a transmitter density $\lambda_{v}$. The channels between the CR transmitters and the primary receiver are called interference channels. For analytical tractability, we assume that the interference channels only consist of path loss. This assumption has been adopted in many system-level studies (e.g., [6], [10], and [22]-[24]), and a recent study [25] has shown that considering fading channels does not cause major changes in the probability distribution function (pdf) of $I_{p}$. The distance between the $j$ th $(1 \leq j<\infty)$ CR transmitter and the primary receiver is denoted by $d_{j},\left(L \leq d_{1} \leq d_{2} \cdots \leq d_{j} \leq\right.$ $\left.\ldots \leq d_{\infty}\right)$. Further assume that all VAAs use the same power $P$. The aggregate interference power at the primary receiver is given by

$$
I_{p}=P \sum_{j=1}^{\infty}\left(d_{j}\right)^{-\alpha}, \quad L \leq d_{1} \leq d_{2} \leq \cdots \leq d_{\infty}
$$

where $\alpha$ is the path-loss exponent. Although the exact spatial distribution of the VAAs may not be a Poisson distribution, it is reasonable to assume that a Poisson point process with the same density $\lambda_{v}$ can be used for calculating the pdf of $I_{p}$. The characteristic function of the random variable $I_{p}$ can then be obtained as [25]

$$
\phi_{I_{p}}(\omega)=\exp \left\{\lambda_{c} \pi L^{2}\left[1-e^{i \omega L^{-\alpha}}\right]+i \omega \lambda_{c} \pi \int_{0}^{L^{-\alpha}} t^{-2 / \alpha} e^{i \omega t} d t\right\} .
$$

From (14), the pdf of $I_{p}$ can numerically be computed by taking the inverse Fourier transform of $\phi_{I_{p}}(\omega)$. However, since this numerical method is not much flexible, analytical approximations are desirable. Previous numerical studies have shown that the pdf of $I_{p}$ is skewed to the left [24], [25]. Therefore, we will subsequently examine the feasibility of using two leftskewed distributions, i.e., lognormal and gamma distributions, to approximate the pdf of $I_{p}$ by fitting the mean and variance. Using the Campbell theorem [26], the mean and variance of $I_{p}$ can be evaluated as [27]

$$
\begin{aligned}
m_{p} & =\mathbb{E}\left(I_{p}\right)=\frac{2 \pi \lambda_{v} P}{\alpha-2} L^{2-\alpha} \\
v_{p} & =\frac{\pi \lambda_{v} P^{2}}{\alpha-1} L^{2-2 \alpha}
\end{aligned}
$$

respectively. The cumulative distribution function (cdf) of a lognormal distribution with a mean parameter $\mu$ and a standard deviation parameter $\sigma$ (evaluated under the natural logarithm of the variable) is given by

$$
F_{\mathrm{LN}}(x ; \mu, \sigma)=\frac{1}{2}+\frac{1}{2} \operatorname{erf}\left(\frac{\ln (x)-\mu}{\sqrt{2} \sigma}\right) .
$$

By matching the mean and variance, the cdf of $I_{p}$ can be approximated by $F_{\mathrm{LN}}\left(x ; \mu_{p}, \sigma_{p}\right)$, where the mean and variance are given by

$$
\begin{aligned}
\mu_{p} & =\frac{1}{2} \ln \left(\frac{m_{p}^{4}}{m_{p}^{2}+v_{p}}\right) \\
\sigma_{p}^{2} & =\ln \left(\frac{m_{p}^{2}+v_{p}}{m_{p}^{2}}\right) .
\end{aligned}
$$

On the other hand, the cdf of a gamma distribution with a shape parameter $k$ and a scale parameter $\theta$ is given by

$$
F_{\mathrm{Ga}}(x ; k, \theta)=\frac{\gamma(k, x / \theta)}{\Gamma(k)}
$$

where $\Gamma(\cdot)$ is the Gamma function, and $\gamma(x, y)$ is the incomplete Gamma function, which is defined as

$$
\gamma(x, y)=\int_{0}^{x} t^{y-1} e^{-t} d t
$$

A gamma random variable having the same mean and variance as $I_{p}$ has a cdf $F_{\mathrm{Ga}}\left(x ; k_{p}, \theta_{p}\right)$ with $k_{p}=m_{p}^{2} / v_{p}$ and $\theta_{p}=$ $v_{p} / m_{p}$. Fig. 5 compares the cdf's of $I_{p}$ calculated by the numerical method [i.e., inverse Fourier transform of (14)] with those obtained from lognormal and gamma approximations. It is observed that, for practical values of $L(L \geq 4)$, both the lognormal and gamma distributions can approximate well the cdf of $I_{p}$, whereas the gamma approximation slightly outperforms the lognormal approximation. Moreover, Fig. 5 confirms that the exclusion-region mechanism is very effective in managing the aggregate interference by implementing different values of the radius $L$. 


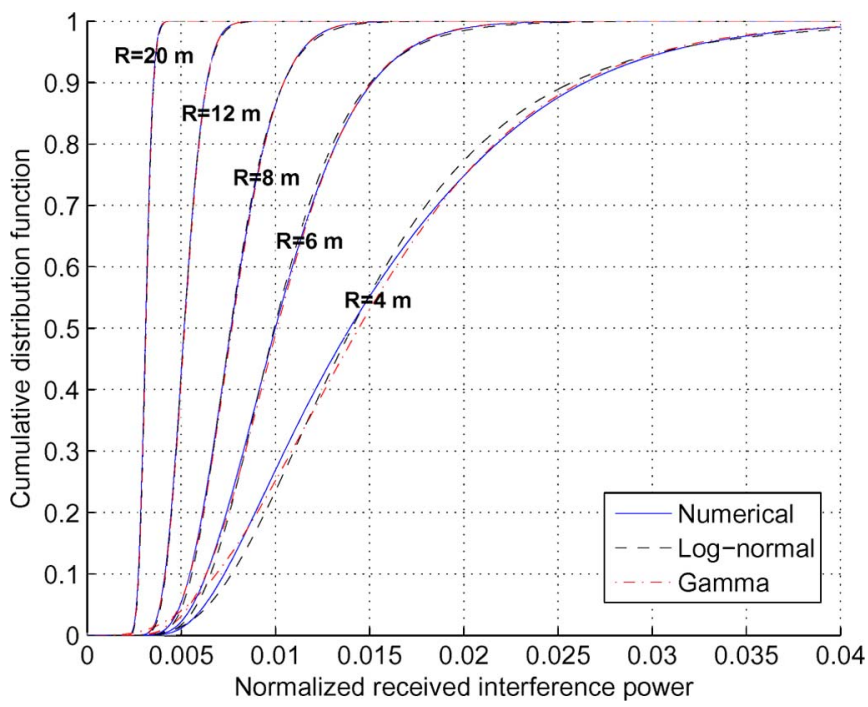

Fig. 5. Cdf's of the normalized interference power $I_{p}$ (normalized to the VAA transmit power $P$ ) from the distributed VAAs to the primary receiver with an exclusion region radius $L\left(\alpha=3\right.$ and $\lambda_{v}=10^{-2}$ users $\left./ \mathrm{m}^{2}\right)$.

Now, we will discuss the mutual interference $I_{s}$ among VAAs. Consider an arbitrary secondary receiver in a VAA. The interfering CR transmitters from other VAAs will be at least $R$ distance away since VAAs cannot overlap. In other words, it appears if the secondary receiver has an exclusion region of radius $R$. Therefore, the previously established results in (13) -(20) can be applied to evaluate $I_{s}$ by simply changing the parameter $L$ to $R$. We note that this method only computes an upper bound of $I_{s}$. A more precise but less-tractable model of $I_{s}$ is possible [24] but will not be pursued in our study.

\section{System Capacity}

The link-level analysis in Section II concerns only one VAA link at a time, and we have quantified the link capacities given fixed VAA antenna numbers $M_{t}$. As illustrated in Fig. 4, the system-level capacity study concerns multiple VAA links. Different VAAs may have different numbers of antennas due to the random locations and availability of cooperating CR users. Therefore, $M_{t}$ should be treated as a random variable at the system level. The (average) system capacity of the hybrid CR network is defined as the sum capacity of various VAA links. It can be calculated as the product of the VAA spatial density and the averaged link capacity, i.e.,

$$
C=\lambda_{v} \sum_{N=1}^{M_{r}} C_{L}(N) f_{M_{t}}(N)
$$

where $C_{L}(\cdot)$ is the ergodic link capacity given by (9), and $f_{M_{t}}(\cdot)$ is the pdf of $M_{t}$. This system capacity measures the average transmitted bits per second per hertz per square meter and has a unit of $\mathrm{b} / \mathrm{s} / \mathrm{Hz} / \mathrm{m}^{2}$. We note that $M_{t} \leq M_{r}$ is assumed in (22). This assumption reflects a common practice that a VAA only seeks to match its antenna number with the receiver side. In other words, at most $M_{r}-1$ cooperating users with the best cooperation-channel qualities are selected in a VAA. Typically, in a hybrid CR network, the licensed band network is an estab- lished network (e.g., a cellular network) with a predetermined capacity, whereas the secondary band is further introduced to the licensed network to expand the system capacity. Therefore, we are particularly interested in the increased system capacity given by

$$
\begin{aligned}
\Delta C & =C-\lambda_{v} C_{L}(1) \\
& =\lambda_{v} \sum_{N=2}^{M_{r}}\left(C_{L}(N)-C_{L}(1)\right) f_{M_{t}}(N) .
\end{aligned}
$$

In (22) and (23), the link capacity $C_{L}(\cdot)$ was studied in Section II. In what follows, we will further investigate $\lambda_{v}$ and $f_{M_{t}}(N)$.

1) VAA Density $\lambda_{v}$ : The point process describing the VAA distribution can be obtained from the point process representing the CR user distribution through thinning operations (i.e., deleting points). The spatial distribution of all CR users is described by a stationary Poisson point process $\Phi_{c}$ with density $\lambda_{c}$. Since two target CR users (i.e., the centers of two VAAs) should be at least $2 R$ distance apart, a new point process $\Phi_{m}$ can be obtained from $\Phi_{c}$ by deleting points that lie closer than $2 R$. This new point process, called the Marten hardcore process, has a density [26, p. 164]

$$
\lambda_{m}=\frac{1-\exp \left(-4 \lambda_{c} \pi R^{2}\right)}{4 \pi R^{2}} .
$$

From $\Phi_{m}$, CR users located within the exclusion regions should further be eliminated, resulting in a new point process $\Phi_{e}$. The percentile of the exclusion region area on the entire plane is given by $[26$, p. 83$]$

$$
\chi_{e}=1-\exp \left(-\lambda_{p} \pi L^{2}\right) .
$$

The density of $\Phi_{e}$ is therefore

$$
\lambda_{e}=\lambda_{m}\left(1-\chi_{e}\right)=\frac{\exp \left(-\lambda_{p} \pi L^{2}\right)\left[1-\exp \left(-4 \lambda_{c} \pi R^{2}\right)\right]}{4 \pi R^{2}} .
$$

Finally, since the VAA density cannot exceed the licensed band user density $\lambda_{l}$, we have

$$
\lambda_{v}=\min \left(\lambda_{e}, \lambda_{l}\right)
$$

2) $P D F f_{M_{t}}(\cdot)$ of $M_{t}$ : The discrete random variable $M_{t}$ denotes the number of antennas in VAAs. The discrete random variable $M_{t}-1$ denotes the number of cooperating users in VAAs. As described in Section II-A, the cooperating users are selected only if the target user can guarantee a minimum SINR $\rho_{\text {cr }}$ at each of them. To obtain the pdf $f_{M_{t}}(\cdot)$, let us first consider the corresponding cdf $F_{M_{t}}(\cdot)$ of $M_{t}$. The value of $F_{M_{t}}(N)$ gives the probability that a VAA has at least $N$ antennas. It follows that the complementary cdf $1-F_{M_{t}}(N)$ means the probability that a VAA has more than $N$ antennas (i.e., at least $N+1$ antennas). Two conditions need to be satisfied for a VAA to have more than $N$ antennas: 1) The distance $d_{N}$ of the $N$ th furthest cooperating user to the target CR user should be smaller than the VAA radius $R$; and 2) the minimum power $P_{N}$ required to support $N$ cooperating users, 
each with a minimum SINR $\rho_{\mathrm{cr}}$, should be less than the maximum allowable power $P$. We then have

$$
1-F_{M_{t}}(N)=F_{d_{N}}(R) F_{P_{N} \mid d_{N} \leq R}(P)
$$

where $F_{d_{N}}(\cdot)$ denotes the cdf of $d_{N}$, and $F_{P_{N} \mid d_{N} \leq R}(\cdot)$ denotes the cdf of $P_{N}$ conditioned on $d_{N} \leq R$. We will subsequently try to solve the right-hand side of (28).

To calculate $F_{d_{N}}(R)$, let us define a new random variable $g_{N}=d_{N}^{2}$. Given that CR users are distributed according to a Poisson field with density $\lambda_{c}, g_{N}$ has an Erlang distribution [26] with a shape parameter $N$ and a rate parameter $\pi \lambda_{c}$. The Erlang cdf with a shape parameter $k$ and a rate parameter $\lambda$ is denoted by $F_{\mathrm{Er}}(x ; k, \lambda)$ and is given by [26]

$$
F_{\mathrm{Er}}(x ; k, \lambda)=1-\sum_{n=0}^{k-1} \frac{e^{-\lambda x^{2}}\left(\lambda x^{2}\right)^{n}}{n !}
$$

where $n$ ! denotes the factorial of $n$. We can then write

$$
F_{d_{N}}(R)=F_{\operatorname{Er}}\left(R^{2} ; N, \pi \lambda_{c}\right) .
$$

Now, let us consider the conditional cdf $F_{P_{N} \mid d_{N} \leq R}(\cdot)$ of $P_{N}$ in (28). As shown in the Appendix, we have

$$
F_{P_{N} \mid d_{N} \leq R}(P)=\frac{1}{2}+\frac{1}{2} \operatorname{erf}\left[\frac{\ln \left(1+G / \rho_{\mathrm{cr}}-N\right)-\mu_{s 0}-\mu_{D}}{\sqrt{2\left(\sigma_{s 0}^{2}+\sigma_{D}^{2}\right)}}\right]
$$

where $G$ is the CDMA spreading/processing gain in the secondary band, and $\mu_{s 0}, \mu_{D}, \sigma_{s 0}$, and $\sigma_{D}$ are defined in the last paragraph of the Appendix. We note that the spreading gain is proportional to the bandwidth of the secondary band. Substituting (30) and (31) into (28), we can express the cdf of $M_{t}$, i.e., the random number of antennas in a VAA, as

$$
\begin{aligned}
F_{M_{t}}(N) & =1-F_{\operatorname{Er}}\left(R^{2} ; \lambda_{c} \pi, N\right) \\
& \times F_{\mathrm{LN}}\left(1+G / \rho_{\mathrm{cr}}-N ; \mu_{s 0}+\mu_{D}, \sqrt{2\left(\sigma_{s 0}^{2}+\sigma_{D}^{2}\right)}\right) .
\end{aligned}
$$

Given $F_{M_{t}}(N)$, the corresponding pdf $f_{M_{t}}(N)$ of the number of antennas in a VAA can be obtained. Finally, combining $f_{M_{t}}(N)$, the link capacity $C_{L}(N)$ given by (9), and VAA density given by (27), the system capacity defined in (22) can be calculated.

\section{Maximum System Capacity}

In Section III-C, we have derived the system capacity of the hybrid CR network as a function of multiple factors, including the primary user density $\lambda_{p}$, CR user density $\lambda_{c}$, licensed channel-user density $\lambda_{l}$, exclusion region radius $L$, VAA radius $R$, VAA transmit power $P$, average SINR at the $\mathrm{BS} \rho_{\mathrm{bs}}$, minimum average SINR at the cooperating users $\rho_{\text {cr }}$, background interference in the secondary band $I_{0}$, and spreading gain $G$. In Section III-B, the interference to primary receivers $I_{p}$ has been derived as a function of $\lambda_{p}, \lambda_{c}, \lambda_{l}, L, R$, and $P$. This interference $I_{p}$ should fulfill certain constraints, such

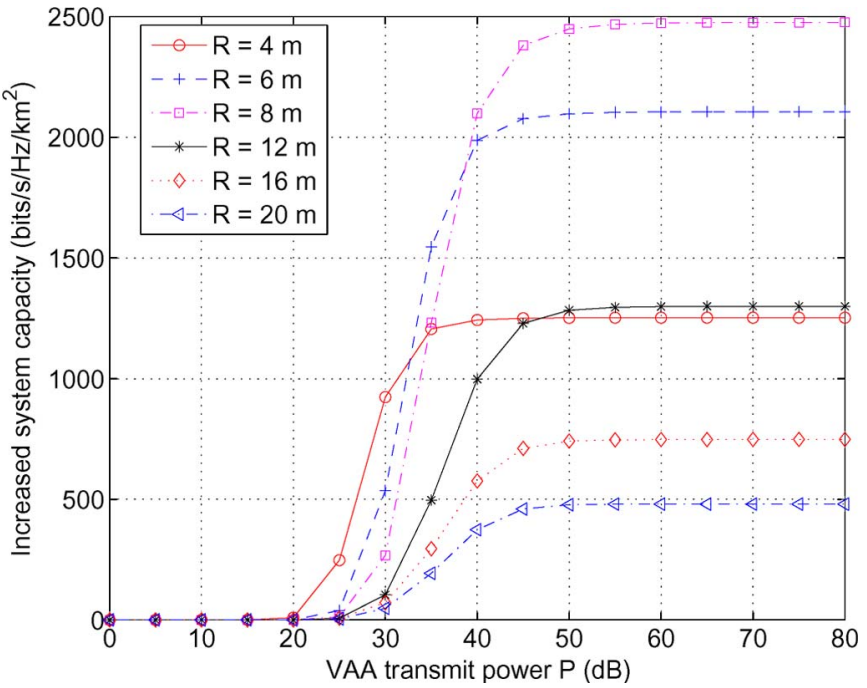

Fig. 6. Increased system capacity $\Delta C$ as a function of the VAA transmit power $P$ in the secondary band with different values of the VAA radius $R\left(\alpha=3, M_{r}=8, \lambda_{p}=10^{-4}\right.$ users $/ \mathrm{m}^{2}, \lambda_{c}=10^{-2}$ users $/ \mathrm{m}^{2}$, $\lambda_{l}=10^{-3}$ users $/ \mathrm{m}^{2}, L=30 \mathrm{~m}, \rho_{\mathrm{bs}}=8 \mathrm{~dB}, \rho_{\mathrm{cr}}=15 \mathrm{~dB}, I_{0}=10$, and $G=10 \mathrm{~dB})$

as peak interference power constraints, average interference power constraints, or interference outage constraints [10], [11]. Mathematically, we can express an interference constraint as $\mathcal{F}_{\text {Cont }}\left(I_{p}\right) \leq I_{\text {lim }}$, where $\mathcal{F}_{\text {Cont }}(\cdot)$ is a constraint-related mapping function and $I_{\text {lim }}$ is a scalar or vector specifying certain constraint values.

In planning a cooperative hybrid CR network, we are interested in the following optimization problem: Given a licensed band network $\left(\lambda_{c}, \lambda_{l}\right.$, and $\left.\rho_{\mathrm{bs}}\right)$ and a secondary band $\left(\lambda_{p}\right.$, $I_{0}$, and $\left.G\right)$ to be used under a certain interference constraint $\left(I_{\text {lim }}\right)$, how can one decide adjustable system parameters $(L$, $R, P$, and $\rho_{\text {cr }}$ ) so that the total system capacity $C$ defined in (22) is maximized? Maximizing $C$ is equivalent to maximizing the increased system capacity $\Delta C$ given by (23). Therefore, the optimization problem can formally be stated as follows: Given $\lambda_{p}, \lambda_{c}, \lambda_{l}, \rho_{\mathrm{bs}}, I_{0}, G$, and $I_{\mathrm{lim}}$, find

$$
\begin{aligned}
\Delta C_{\max }= & \max _{L, R, P, \rho_{\mathrm{cr}}} \Delta C\left(L, R, P, \rho_{\mathrm{cr}}\right) \\
& \text { s.t. } \quad \mathcal{F}_{\text {Cont }}\left(I_{p}\right) \leq I_{\text {lim }} L>0 R>0 P>0 \rho_{\mathrm{cr}}>0
\end{aligned}
$$

where $\Delta C_{\max }$ denotes the maximum increased system capacity. There are complex tradeoffs involved in finding the optimal parameter values. For example, if $\rho_{\text {cr }}$ increases, the link capacity also increases, but the average antenna number in a VAA reduces. For another example, if $R$ increases, the VAA density reduces, but the average number of antennas in a VAA increases. To illustrate some of these tradeoffs, in Fig. 6, we show $\Delta C$ as a function of $P$ with different values of $R$. With the given parameter set, it is found that $R=8 \mathrm{~m}$ gives the maximum capacity when $P$ is sufficiently high. Moreover, at high values of $P$, the capacities saturate to certain limits because the VAA performance becomes limited by the mutual interference $I_{s}$ among VAAs. 


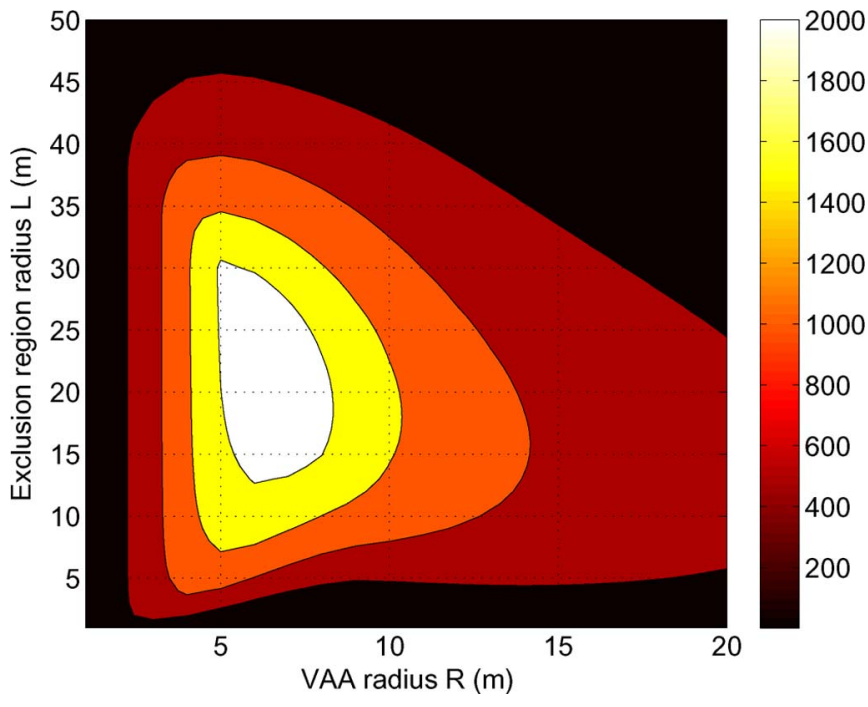

Fig. 7. Increased system capacity $\Delta C$ as a function of the exclusion region radius $L$ and VAA radius $R\left(\alpha=3, M_{r}=8, \lambda_{p}=10^{-4}\right.$ users $/ \mathrm{m}^{2}, \lambda_{c}=$ $10^{-2}$ users $/ \mathrm{m}^{2}, \lambda_{l}=10^{-3}$ users $/ \mathrm{m}^{2}, \rho_{\mathrm{bs}}=8 \mathrm{~dB}, \rho_{\mathrm{cr}}=15 \mathrm{~dB}, I_{0}=1$, $G=20 \mathrm{~dB}$, and $I_{\lim }=1$ ).

In what follows, we will solve the optimization problem in (33) under an average interference power constraint given as $\mathbb{E}\left(I_{p}\right) \leq I_{\text {lim }}$, where $\mathbb{E}\left(I_{p}\right)$ can be calculated from (15). To reveal more insight into the impact of $\rho_{\text {cr }}$ on $\Delta C_{\text {max }}$, we will study $\Delta C_{\max }$ as a function of $\rho_{\mathrm{cr}}$. As shown in Fig. 6, we find that $\Delta C$ is a monotonically increasing function on $P$. This suggests that the maximum capacity is achieved if the maximum allowable power $P$ is used. Therefore, we can take the equality in the average interference constraint and compute $P$ from other parameters based on (15). The new optimization problem under the average interference power constraint can then be stated as follows: Given $\lambda_{p}, \lambda_{c}, \lambda_{l}, \rho_{\mathrm{bs}}, I_{0}, G$, and $I_{\mathrm{lim}}$, find

$$
\begin{aligned}
\Delta C_{\max }\left(\rho_{\mathrm{cr}}\right)= & \max _{L, R} \Delta C\left(L, R, P, \rho_{\mathrm{cr}}\right) \\
\text { s.t. } & P=\frac{(\alpha-2) I_{\text {lim }}}{2 \pi L^{2-\alpha} \lambda_{v}} L>0 R>0 \rho_{\mathrm{cr}}>0 .
\end{aligned}
$$

With $\rho_{\mathrm{cr}}=15 \mathrm{~dB}$, in Fig. 7, we illustrate $\Delta C$ as a function of $R$ and $L$. It is observed that $\Delta C(R, L)$ forms a concave surface. Although a strict proof on the concavity of $\Delta C$ on $(R, L)$ is difficult, through simulations, we have found that the concavity is preserved in all cases with practical parameter values. Therefore, (34) can be solved as a concave optimization problem, and standard convex searching algorithms [28] can be used to find the maximum capacity $\Delta C_{\max }$.

\section{NUMERICAL RESUlTS AND Discussions}

This section aims to numerically evaluate the system capacity of the proposed cooperative hybrid CR networks. Specifically, we consider a cellular network [e.g., Universal Mobile Telecommunications System (UMTS)] as the licensed network that aims to expand its capacity by immigrating to a hybrid $\mathrm{CR}$ network. The secondary band is acquired from the FMC spectrum. FMC systems (e.g., fixed WiMax) are characterized

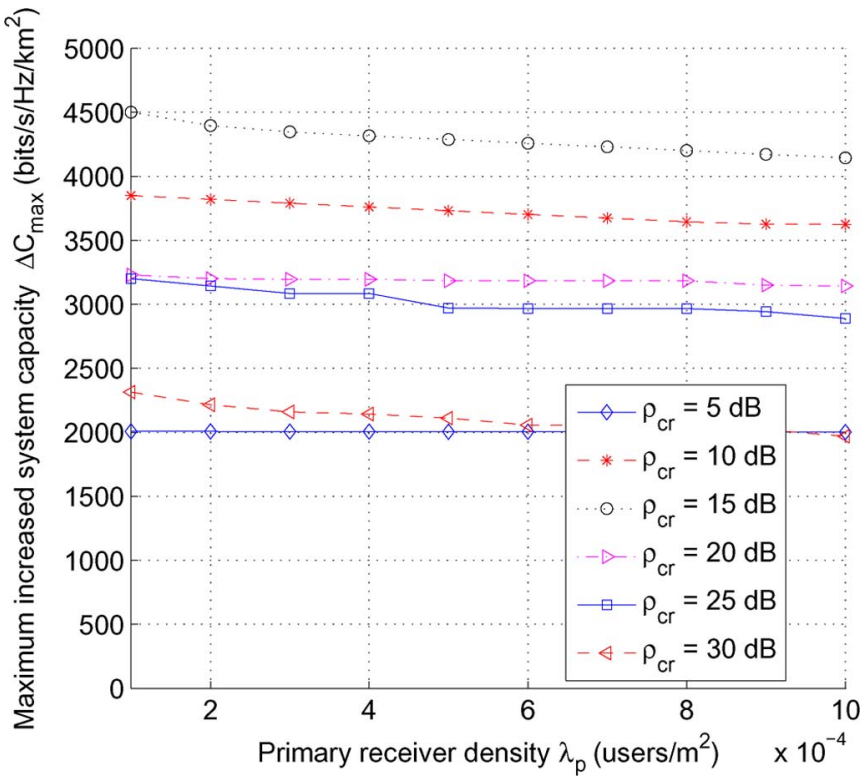

Fig. 8. Maximum increased system capacity $\Delta C_{\max }$ as a function of the primary user density $\lambda_{p}$ with different values of $\rho_{\mathrm{cr}}\left(\alpha=3, M_{r}=8, \lambda_{c}=\right.$ $10^{-2}$ users $/ \mathrm{m}^{2}, \lambda_{l}=10^{-3}$ users $/ \mathrm{m}^{2}, \rho_{\mathrm{bs}}=8 \mathrm{~dB}, I_{0}=1, G=20 \mathrm{~dB}$, and $\left.I_{\lim }=1\right)$.

by highly directive transmissions that, intuitively, present opportunities for secondary networks to share their spectrum [29]. It is interesting to note that interweave CR networks may not be able to properly operate in these bands because the directive primary transmissions are difficult to be detected. Therefore, interweave CR networks can easily cause harmful interference to hidden primary receivers. On the contrary, overlay CR networks can still exploit the FMC bands by directly focusing on the primary receivers. In this paper, an average interference power constraint is assumed to protect the FMC receivers. The performance gain of deploying the hybrid CR network is measured by the maximum increased system capacity $\Delta C_{\max }\left(\rho_{\text {cr }}\right)$ given by (34). Unless otherwise stated, we assume $\alpha=3, M_{r}=8, \lambda_{p}=$ $10^{-4}$ users $/ \mathrm{m}^{2}, \lambda_{l}=10^{-3}$ users $/ \mathrm{m}^{2}, \lambda_{c}=10^{-2}$ users $/ \mathrm{km}^{2}$, $\rho_{\mathrm{bs}}=8 \mathrm{~dB}, I_{0}=1, I_{\mathrm{lim}}=1$, and $G=20 \mathrm{~dB}$. All power values are normalized to the noise power in the secondary band. The increased capacity of the cooperative hybrid CR network over the traditional licensed network will be evaluated at discrete values of $\rho_{\mathrm{cr}}(5-30 \mathrm{~dB}$ with $5-\mathrm{dB}$ increments) and presented in the unit of $\mathrm{b} / \mathrm{s} / \mathrm{Hz} / \mathrm{km}^{2}$.

Fig. 8 shows the impact of the primary user density $\lambda_{p}$ on the increased capacity $\Delta C_{\max }\left(\rho_{\mathrm{cr}}\right)$. It is observed that even a ten times increase of $\lambda_{p}$ only results in insignificant reductions of the capacity results. In Fig. 9, $\Delta C_{\max }\left(\rho_{\text {cr }}\right)$ is shown as a function of $I_{0}$, which represents the interference from the primary (FMC) system to the distributed VAA network. Similar to Fig. 8, the capacity results are only slightly reduced when $I_{0}$ increases ten times. Figs. 8 and 9 demonstrate that the capacity improvement of a cooperative hybrid CR network is insensitive to the characteristics of the primary network. This immunity to the primary system represents a fundamental advantage of the cooperative hybrid CR network over the noncooperative hybrid CR network, whose capacity improvement (i.e., the capacity of pure CR network) can greatly fluctuate with small changes 


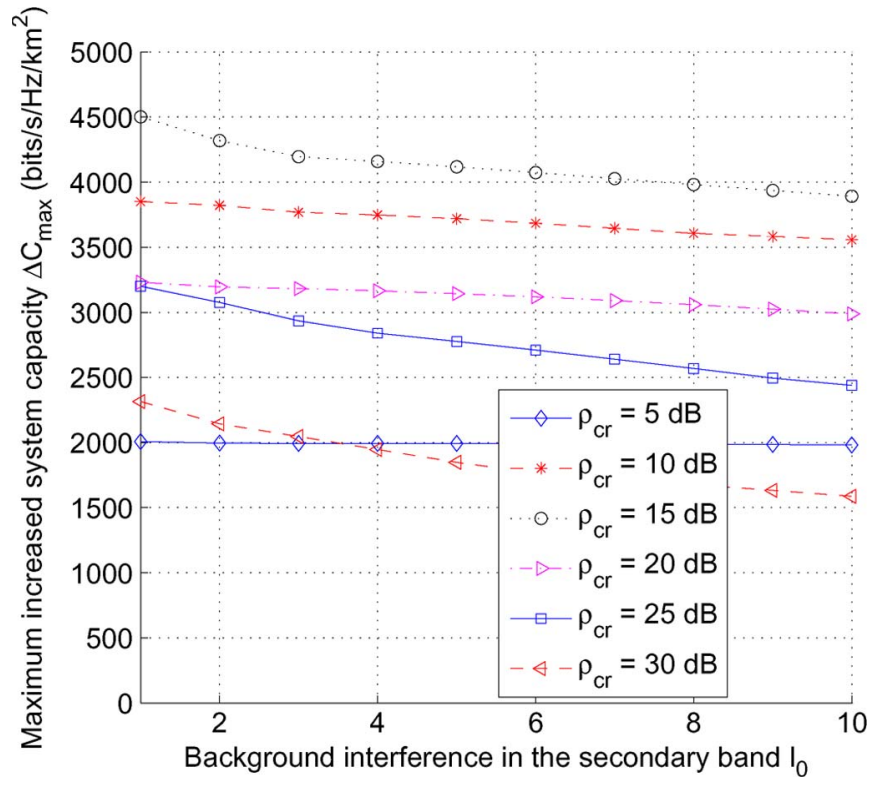

Fig. 9. Maximum increased system capacity $\Delta C_{\max }$ as a function of $I_{0}$ with different values of $\rho_{\mathrm{cr}}\left(\alpha=3, M_{r}=8, \lambda_{p}=10^{-4}\right.$ users $/ \mathrm{m}^{2}$, $\lambda_{c}=10^{-2}$ users $/ \mathrm{m}^{2}, \lambda_{l}=10^{-3}$ users $/ \mathrm{m}^{2}, \rho_{\mathrm{bs}}=8 \mathrm{~dB}, G=20 \mathrm{~dB}$, and $\left.I_{\lim }=1\right)$.

in the primary system [10]-[13]. For example, in [11], it was found that the capacity is reduced by a factor of 100 when $\lambda_{p}$ is increased by a factor of 10 . Moreover, in cooperative hybrid CR networks, the capacity improvement is not affected by the cell radius (communication range). This is because the VAAs usually have enough power allowance in the licensed band to compensate for the propagation loss and maintain a fixed SINR $\rho_{\text {bs }}$ at the BS (as in a UMTS system). On the contrary, in noncooperative networks, the capacity improvement quickly diminishes with the increasing cell radius [10], [11].

In Fig. 10, $\Delta C_{\max }\left(\rho_{\mathrm{cr}}\right)$ is computed as a function of the $\mathrm{CR}$ user density $\lambda_{c}$. The impact of $\lambda_{c}$ on $\Delta C_{\max }\left(\rho_{\mathrm{cr}}\right)$ is shown to be significant. The highest capacity is given by $\rho_{\mathrm{cr}}=15 \mathrm{~dB}$, where a nearly linear relationship is observed between $\lambda_{c}$ and $\Delta C_{\max }\left(\rho_{\text {cr }}\right)$. This result suggests that the hybrid CR network with distributed VAAs is best deployed in urban areas where the user density is sufficiently high. This conclusion agrees with our previous study in [6].

Finally, Fig. 11 shows $\Delta C_{\max }\left(\rho_{\mathrm{cr}}\right)$ as a function of the spreading gain $G$. It is observed that a high spreading gain can effectively enhance the system capacity. We note that the spreading gain is roughly proportional to the bandwidth of the secondary band. A high spreading gain is feasible in practice since the secondary band usually has a wide bandwidth. For example, if the licensed UMTS band with a 5-MHz bandwidth is shared by 20 users and the secondary band has a $25-\mathrm{MHz}$ bandwidth, the processing gain can roughly be estimated as $G=25 / 5 \times 20=100=20 \mathrm{~dB}$. Compared with other multiuser schemes, such as the FDMA studied in [6], the CDMA scheme provides a clear benefit since it can naturally turn the wideband advantage of the secondary band into a SINR gain in the VAA signaling scheme to achieve better system performance. From another perspective, Fig. 11 also indicates the advantage of the cooperative hybrid CR network

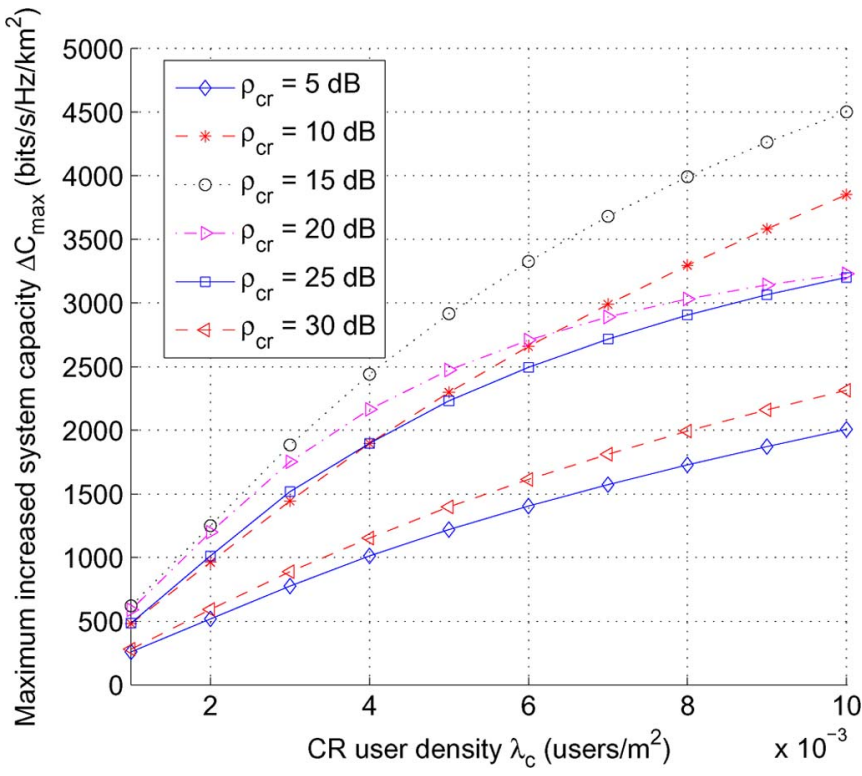

Fig. 10. Maximum increased system capacity $\Delta C_{\max }$ as a function of the CR user density $\lambda_{c}$ with different values of $\rho_{\mathrm{cr}}\left(\alpha=3, M_{r}=8, \lambda_{p}=\right.$ $10^{-4}$ users $/ \mathrm{m}^{2}, \lambda_{l}=10^{-3}$ users $/ \mathrm{m}^{2}, \rho_{\mathrm{bs}}=8 \mathrm{~dB}, I_{0}=1, G=20 \mathrm{~dB}$, and $\left.I_{\lim }=1\right)$.

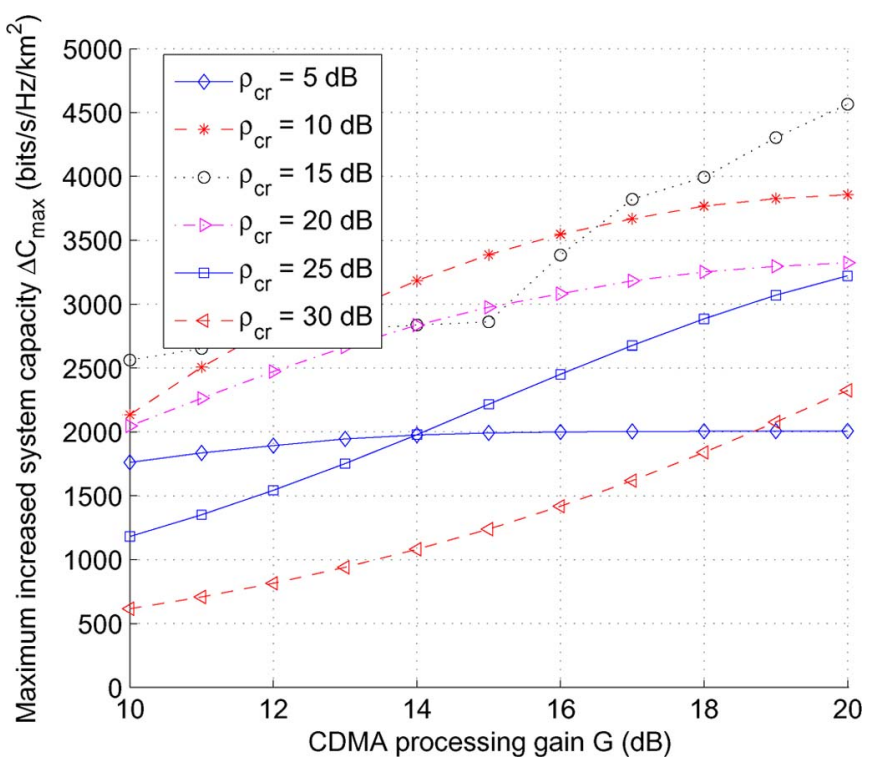

Fig. 11. Maximum increased system capacity $\Delta C_{\max }$ as a function of the processing gain $G$ with different values of $\rho_{\mathrm{cr}}\left(\alpha=3, M_{r}=8, \lambda_{p}=\right.$ $10^{-4}$ users $/ \mathrm{m}^{2}, \lambda_{c}=10^{-2}$ users $/ \mathrm{m}^{2}, \lambda_{l}=10^{-3}$ users $/ \mathrm{m}^{2}, \rho_{\mathrm{bs}}=8 \mathrm{~dB}$, $I_{0}=1$, and $I_{\lim }=1$ ).

over conventional cooperative licensed networks, which use a fraction of the licensed spectrum to enable cooperation. Clearly, conventional cooperative licensed networks can only have a small spreading gain. In addition, it is usually difficult to guarantee that the advantages of cooperative communications outweigh the disadvantages of wasting a fraction of the licensed bandwidth to enable cooperation.

For comparison purposes, we note that the system capacity of the licensed cellular network with the default parameter values is given by $\lambda_{l} \bar{C}_{L}(1)=5160 \mathrm{~b} / \mathrm{s} / \mathrm{Hz} / \mathrm{km}^{2}$. In Figs. $8-10$, the highest capacity improvements are shown to be almost equal to the capacity of the original licensed cellular system. 
We note that this capacity improvement is obtained under the assumption of using only one secondary band and a very tight interference constraint $I_{\lim }=1$, which limits the average interference power received at the primary receiver to be at the same level of the background noise. When multiple secondary bands are available or the interference constraint is not so tight, the proposed cooperative hybrid CR network can achieve even higher capacity improvements, making it a promising solution for future wireless communication networks.

\section{CONCLusion}

We have proposed a cooperative hybrid CR network that simultaneously operates on a dedicated licensed band and a secondary band. It has been assumed that randomly located CR users can cooperate in the secondary band to form distributed VAAs, which are then used to facilitate communications in the licensed band utilizing virtual MIMO technologies. We have first presented a novel VAA signaling scheme that employs power control to prevent harmful noise propagation. The resulting virtual MIMO link capacity has been derived as a function of the received SINRs in the secondary and licensed bands. Extending to the multiuser case, we have subsequently analyzed the system capacity, taking into account multiple factors, such as the primary user density, CR user density, primary exclusion region radius, VAA radius, etc. The maximum system capacity has been formulated as an optimization problem and solved under an average interference power constraint. Numerical results have indicated that the proposed cooperative hybrid CR network can achieve a promising capacity improvement when high CR user densities and a wide secondary bandwidth are available. More importantly, this capacity improvement is insensitive to the primary user density and interference power from the primary network. We conclude that the proposed cooperative hybrid CR network is a promising alternative to conventional pure $\mathrm{CR}$ networks to be deployed in urban scenarios.

\section{APPENDIX}

\section{DERIVATION OF (31)}

The total power $P_{N}$ can be written as the sum of the power allocated to each cooperating user, i.e.,

$$
P_{N}=\sum_{n=1}^{N} P_{n}
$$

where $P_{n}$ denotes the power allocated to the $n$th user. For simplicity, we consider only the path loss in the propagation channels from the target CR user to cooperating users. This assumption can be justified for the following two reasons: 1) The VAA is assumed to be "local," and therefore, shadowing is expected to have a small impact on the channel gain variations; and 2) a wideband CDMA signaling scheme is assumed so that the small-scale fading effect is averaged over the wide bandwidth. The minimum SINR requirement at a cooperating user can be expressed as

$$
\frac{G P_{n}\left(d_{n}\right)^{-\alpha}}{\left(P_{N}-P_{n}\right) d_{n}^{-\alpha}+I_{s}+I_{0}} \geq \rho_{\mathrm{cr}}, \quad(n=1,2, \ldots, N)
$$

where $G$ is the CDMA processing gain (spreading gain), $d_{n}$ is the distance from the $n$th cooperating user to the target user, $\alpha$ is the path loss exponent, $I_{s}$ is the mutual interference from other VAAs, and $I_{0}$ is background interference. The mutual interference term $I_{s}$ is assumed to be the same at all cooperating users in a VAA. This assumption is reasonable because a VAA operates in a local area. The term $\left(P_{N}-P_{n}\right) d_{n}^{-\alpha}$ in (36) represents multiuser interference within a VAA. From (35) and (36), we get

$$
\begin{aligned}
F_{P_{N} \mid d_{N} \leq R}(P) & =\mathbb{P}\left(P_{N} \leq P\right) \\
& =\mathbb{P}\left(I_{s 0} D_{N} \leq 1+G / \rho_{\mathrm{cr}}-N\right)
\end{aligned}
$$

where the operator $\mathbb{P}(x)$ returns the probability that statement $x$ is true, $I_{s 0}=\left(I_{s}+I_{0}\right) / P$, and $D_{N}=\sum_{n=1}^{N}\left(d_{n}\right)^{\alpha}$. Our focus is now on obtaining the cdf's of the two independent random variables $I_{s 0}$ and $D_{N}$ in (37). Since $I_{0}$ and $P$ are constants, $I_{s 0}$ is a shifted and scaled version of another random variable $I_{s}$. Therefore, similar to $I_{s}$ (see Section III-B), the cdf of $I_{s 0}$ can also be approximated by lognormal or Nakagami distributions. The mean and variance of $I_{s 0}$ required to perform such approximations are given by

$$
\begin{aligned}
m_{s 0} & =\frac{2 \pi \lambda_{v}}{\alpha-2} L^{2-\alpha}+I_{0} / P \\
v_{s 0} & =\frac{\pi \lambda_{v}}{\alpha-1} L^{2-2 \alpha}
\end{aligned}
$$

respectively. Now, consider the second random variable $D_{N}$ in (37). For $N=2$, the cdf of $D_{N}$ can easily be derived in a closed form, which is given by

$$
F_{D_{1}}(x)=\frac{1-e^{-\lambda_{c} \pi x^{\alpha / 2}}}{1-e^{-\lambda_{c} \pi R^{2}}} \quad\left(0 \leq x \leq R^{\alpha}\right) .
$$

For $N \geq 3$, closed-from expressions for the cdf's are not available, but we can derive the moment-generating function (MGF) $\phi_{D_{N}}(s)$ of $D_{N}$. Since the cooperating CR users are distributed in a Poisson field with density $\lambda_{c}, g_{n}=\left(d_{n}\right)^{2}, g_{n}$ forms a Poisson process with rate $\lambda_{c} \pi$ [26]. The MGF of $D_{N}$ is given by

$$
\begin{aligned}
\phi_{D_{N}}(s) & =\mathbb{E}\left\{e^{s D_{N}}\right\}=\mathbb{E}\left\{e^{s\left(g_{N}\right)^{\alpha / 2}} e^{s \sum_{n=1}^{N-1}\left(g_{n}\right)^{\alpha / 2}}\right\} \\
& =\mathbb{E}_{g_{N}}\left\{e^{s x^{\alpha / 2}} \mathbb{E}\left[\left.e^{s \sum_{n=1}^{N-1}\left(g_{n}\right)^{\alpha / 2}}\right|_{g_{N}=x}\right]\right\}
\end{aligned}
$$

In (41), the first expectation $\mathbb{E}_{g_{N}}\{\cdot\}$ is taken over $g_{N}$, and the second expectation is taken over $g_{n}(1 \leq n \leq N-1)$ conditioned on that $g_{N}=x$. Based on the properties of the Poisson process [26], it is straightforward to obtain the pdf of $g_{N}$ as

$$
f_{g_{N}}(x)=\frac{\left(\lambda_{c} \pi\right)^{N} x^{N-1} e^{-\lambda_{c} \pi x}}{(N-1) ! F_{\operatorname{Er}\left(R^{2} ; \lambda_{c} \pi, N\right)}}, \quad\left(0 \leq x \leq R^{2}\right)
$$


Moreover, given $g_{N}=x, g_{n}$ become independent random variables uniformly distributed from 0 to $x$. We can now evaluate the two expectations in (41) to give

$$
\phi_{D_{N}}(s)=\int_{0}^{R^{2}} f_{g_{N}}(x) e^{s x^{\alpha / 2}}\left[\int_{0}^{x} \frac{1}{x} e^{s y^{\alpha / 2}} d y\right]^{N-1} d x .
$$

Substituting (42) into (43), after some simplifications, we can get

$$
\begin{array}{r}
\phi_{D_{N}}(s)=\frac{\left(\lambda_{c} \pi\right)^{N}}{(N-1) ! F_{\mathrm{Er}}\left(R^{2} ; N, \pi \lambda_{c}\right)} \int_{0}^{R^{2}} e^{-\lambda_{c} \pi x+s x^{\alpha / 2}} \\
\times\left[\int_{0}^{x} e^{s y^{\alpha / 2}} d y\right]^{N-1} d x
\end{array}
$$

where $F_{\mathrm{Er}}(\cdot)$ is the Erlang cdf function given by (29). From (44), numerical inversion methods can be used to calculate the pdf of $D_{N}$ with a high computational intensity.

Inspired by the interference approximation in Section III-B, we would like to see if certain approximations can also be made for the cdf of $D_{N}$. From (44), it is straightforward to obtain the corresponding first and second derivatives $\phi_{D_{N}}^{\prime}(s)$ and $\phi_{D_{N}}^{\prime \prime}(s)$ of the MGF. Let $s=0$ in $\phi_{D_{N}}^{\prime}(s)$ and $\phi_{D_{N}}^{\prime \prime}(s)$, we can obtain the first and second moments of $D_{N}$ as

$$
\begin{aligned}
m_{D}= & \left(1+\frac{2 N-2}{2+a f a}\right) \frac{\left(\lambda_{c} \pi\right)^{-\alpha / 2}}{(N-1) ! F_{\mathrm{Er}}\left(R^{2} ; N, \lambda_{c} \pi\right)} \\
& \times \gamma\left(\lambda_{c} \pi R^{2}, N+\alpha / 2\right) \\
m_{2 D}= & K_{D} \frac{\left(\lambda_{c} \pi\right)^{-\alpha}}{(N-1) ! F_{\mathrm{Er}}\left(R^{2} ; N, \lambda_{c} \pi\right)} \gamma\left(\lambda_{c} \pi R^{2}, N+\alpha\right)
\end{aligned}
$$

respectively, where $K_{D}$ is a constant given by

$$
K_{D}=1+\frac{4(N-1)}{2+\alpha}+\frac{4(N-1)(N-2)}{(2+\alpha)^{2}}+\frac{N-1}{1+\alpha} .
$$

From (45) and (46), the variance of $D_{N}$ can be obtained as $v_{D}=m_{2 D}-\left(m_{D}\right)^{2}$. Numerical inversions have shown that the pdf of $D_{N}$ is skewed to the left. Therefore, lognormal and gamma distributions are used to approximate the cdf of $D_{N}$. Fig. 12 shows the approximation results with $N$ ranging from 2 to 8 . It is observed that the gamma distribution closely matches to the cdf's obtained from numerical methods, whereas the lognormal distribution is less accurate but still yields acceptable performance.

From (38)-(47), we have established that the cdf's of $I_{s 0}$ and $D_{N}$ can reasonably be approximated by gamma or lognormal distributions. We can now return to (37) to calculate the probability $\mathbb{P}\left(I_{s 0} D_{N} \leq 1+G / \rho_{\mathrm{cr}}-N\right)$. The gamma approximation can give better accuracy, whereas the lognormal approximation can give a desirable closed-form formula with slightly reduced accuracy. For analytical convenience, the lognormal approximation will be used in our subsequent analysis. Let us denote the approximated lognormal cdf's for $I_{s 0}$ and

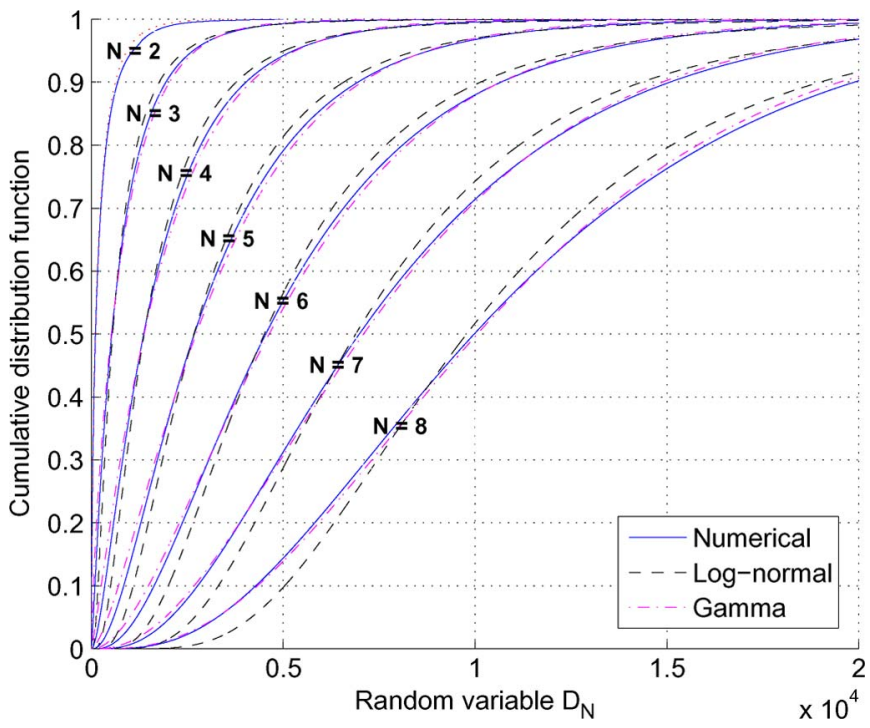

Fig. 12. Cdf's of the random variable $D_{N}$ with different values of the VAA antenna number $N\left(\alpha=3, \lambda_{c}=10^{-2}\right.$ users $/ \mathrm{m}^{2}$, and $\left.R=20 \mathrm{~m}\right)$.

$D_{N}$ by $F_{\mathrm{LN}}\left(x ; \mu_{s 0}, \sigma_{s 0}\right)$ and $F_{\mathrm{LN}}\left(x ; \mu_{D}, \sigma_{D}\right)$, respectively, where the parameters $\mu_{s 0}, \sigma_{s 0}, \mu_{D}$, and $\sigma_{D}$ can be calculated using (18) and (19) with corresponding mean and variance values given by (38)-(46). It follows that the cdf of the product $I_{s 0} D_{N}$ also has a lognormal approximation $F_{\mathrm{LN}}\left(x ; \mu_{s 0}+\right.$ $\left.\mu_{D}, \sqrt{\sigma_{s 0}^{2}+\sigma_{D}^{2}}\right)$. Applying the lognormal cdf function given by (17) to the last expression in (37) gives the conditional cdf in (31).

\section{REFERENCES}

[1] FCC Spectrum Policy Task Force, Report of the spectrum efficiency working group, Nov. 2002. [Online]. Available: http://www. fcc.gov/sptf/reports.html

[2] I. F. Akyildiz, W. Y. Lee, M. C. Vuran, and S. Mohanty, "Next generation dynamic spectrum access/cognitive radio wireless networks: A survey," Comput. Netw., vol. 50, no. 13, pp. 2127-2159, Sep. 2006.

[3] S. Haykin, "Cognitive radio: Brain-empowered wireless communications," IEEE J. Sel. Areas Commun., vol. 23, no. 2, pp. 201-220, Feb. 2005.

[4] X. Hong, Z. Chen, C.-X. Wang, S. Vorobroy, and J. S. Thompson, "Cognitive radio networks: Interference cancellation and management techniques," IEEE Veh. Technol. Mag., vol. 4, no. 4, pp. 76-84, Nov. 2009.

[5] X. Hong, C.-X. Wang, H.-H. Chen, and Y. Zhang, "Secondary spectrum access networks: Recent developments on the spatial models," IEEE Veh. Technol. Mag., vol. 4, no. 2, pp. 36-43, Jun. 2009.

[6] C.-X. Wang, X. Hong, H.-H. Chen, and J. S. Thompson, "On capacity of cognitive radio networks with average interference power constraints," IEEE Trans. Wireless Commun., vol. 8, no. 4, pp. 1620-1625, Apr. 2009.

[7] C. Stevenson, G. Chouinard, Z. Lei, W. Hu, S. Shellhammer, and W. Caldwell, "IEEE 802.22: The first cognitive radio wireless regional area network standard," IEEE Commun. Mag., vol. 47, no. 1, pp. 130138, Jan. 2009.

[8] S. Mangold, A. Jarosch, and C. Monney, "Operator assisted cognitive radio and dynamic spectrum assignment with dual beacons-Detailed evaluation," in Proc. ICST COMSWARE, Delhi, India, Jan. 2006, pp. 1-6.

[9] B. Wild and K. Ramchandran, "Detecting primary receivers for cognitive radio applications," in Proc. IEEE DySPAN, Baltimore, MD, Nov. 2005, pp. $124-130$.

[10] C.-X. Wang, H.-H. Chen, X. Hong, and M. Guizani, "Cognitive radio network management: Tuning into real-time conditions," IEEE Veh. Technol. Mag., vol. 3, no. 1, pp. 28-35, Mar. 2008.

[11] X. Hong, C.-X. Wang, and J. S. Thompson, "Uplink cell capacity of cognitive radio networks under peak interference power constraints," in Proc. IEEE ICCCAS, Xiamen, China, May 2008, pp. 372-377.

[12] J. Gao, H. A. Suraweera, M. Shafi, and M. Faulkner, "Channel capacity of a cognitive radio network in GSM uplink band," in Proc. IEEE ISCIT, Sydney, Australia, Oct. 2007, pp. 1511-1515. 
[13] N. Devroye, M. Vu, and V. Tarokh, "Cognitive radio networks," IEEE Signal Process. Mag., vol. 25, no. 6, pp. 12-23, Nov. 2008.

[14] A. Nosratinia, T. E. Hunter, and A. Hedayat, "Cooperative communication in wireless networks," IEEE Commun. Mag., vol. 42, no. 10, pp. 74-80, Oct. 2004.

[15] M. Dohler, J. Dominguez, and H. Aghvami, "Link capacity analysis for virtual antenna arrays," in Proc. IEEE VTC-Fall, Vancouver, BC, Canada, Sep. 2002, pp. 440-443.

[16] A. Paulraj, R. Nabar, and D. Gore, Introduction to Space-Time Wireless Communications. Cambridge, U.K.: Cambridge Univ. Press, 2003.

[17] M. O. Hasna and M. S. Alouini, "Performance analysis of two-hop relayed transmission over Rayleigh fading channels," in Proc. IEEE VTC-Fall, Vancouver, BC, Canada, Sep. 2002, pp. 24-28.

[18] R. R. Gejji, "Forward-link-power control in CDMA cellular systems," IEEE Trans. Veh. Technol., vol. 41, no. 4, pp. 532-536, Nov. 1992.

[19] C.-X. Wang, X. Hong, X. Ge, X. Cheng, G. Zhang, and J. S. Thompson, "Cooperative MIMO channel models: A survey," IEEE Commun. Mag., vol. 48, no. 2, pp. 80-87, Feb. 2010.

[20] C.-X. Wang, X. Cheng, and D. I. Laurenson, "Vehicle-to-vehicle channel modeling and measurements: Recent advances and future challenges," IEEE Commun. Mag., vol. 47, no. 11, pp. 96-103, Nov. 2009.

[21] X. Cheng, C.-X. Wang, D. I. Laurenson, S. Salous, and A. V. Vasilakos, "An adaptive geometry-based stochastic model for non-isotropic MIMO mobile-to-mobile channels," IEEE Trans. Wireless Commun., vol. 8, no. 9, pp. 4824-4835, Sep. 2009.

[22] E. S. Sousa and J. A. Silvester, "Optimum transmission range in a directsequence spread-spectrum multihop pack radio network," IEEE J. Sel. Areas Commun., vol. 8, no. 5, pp. 762-771, Jun. 1990.

[23] E. S. Sousa, "Performance of a spread spectrum packet radio network link in a Poisson field of interferers," IEEE Trans. Inf. Theory, vol. 38, no. 6, pp. 1743-1754, Nov. 1992.

[24] R. Menon, R. M. Buehrer, and J. H. Reed, "Impact of exclusion region and spreading in spectrum-sharing ad hoc networks," in Proc. 1st Int. Workshop Technol. Policy Accessing Spectr, Boston, MA, Aug. 2006.

[25] X. Hong, C.-X. Wang, and J. S. Thompson, "Interference modeling of secondary networks in spectrum sharing systems," in Proc. IEEE VTCSpring, Singapore, May 2008, pp. 1851-1855.

[26] D. Stoyan, W. S. Kendall, and J. Mecke, Stochastic Geometry and Its Applications. Chichester, U.K.: Wiley, 1986.

[27] S. P. Weber, X. Yang, J. G. Andrews, and G. de Veciana, "Transmission capacity of wireless ad hoc networks with outage constraints," IEEE Trans. Inf. Theory, vol. 51, no. 12, pp. 4091-4102, Dec. 2005.

[28] S. Boyd and L. Vandenberghe, Convex Optimization. Cambridge, U.K.: Cambridge Univ. Press, 2004.

[29] R. Fantacci, D. Marabissi, and D. Tarchi, "Proposal of a fixed communication system sharing the bandwidth of an existing personal communication network," IEEE Trans. Veh. Technol., vol. 57, no. 1, pp. 180-187, Jan. 2008.

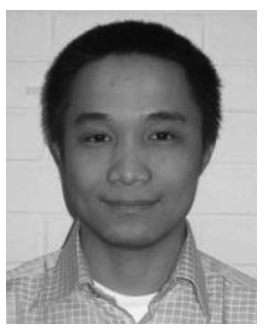

Xuemin Hong received the B.Sc. degree in communication engineering from Beijing Information Science and Technology University, Beijing, China, in 2004 and the Ph.D. degree in wireless communications from Heriot-Watt University, Edinburgh, U.K., in 2008.

Since August 2009, he has been a Postdoctoral Research Associate with the Joint Research Institute for Signal and Image Processing, Heriot-Watt University, where he is also the Network Manager of the "U.K.-China Science Bridges: R\&D on (B) $4 \mathrm{G}$ Wireless Mobile Communications". From January 2009 to July 2009, he was a Postdoctoral Research Fellow with the Department of Electrical and Computer Engineering, University of Waterloo, Waterloo, ON, Canada. From 2004 to 2005, he was affiliated with the Centre for Telecommunication Research, King's College London, London, U.K. He has published 18 technical papers in major international journals and conference proceedings and one book chapter in the area of wireless communications. His research interests include multipleinput-multiple-output and cooperative systems, ultrawideband systems, wireless radio channel modeling, cognitive radio networks, and $\mathrm{B} 4 \mathrm{G}$ wireless communications.

Dr. Hong served as a Publicity Chair for the 2010 IEEE International Conference on Communications and Mobile Computing and as a Technical Program Committee Member for several international conferences.

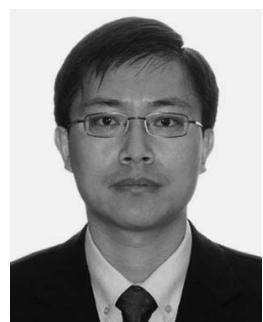

Cheng-Xiang Wang (S'01-M'05-SM'08) received the B.Sc. and M.Eng. degrees in communication and information systems from Shandong University, Shandong, China, in 1997 and 2000, respectively, and the Ph.D. degree in wireless communications from Aalborg University, Aalborg, Denmark, in 2004.

$\mathrm{He}$ has been with Heriot-Watt University, Edinburgh, U.K., since 2005, first as a Lecturer and then as a Reader in 2009. He is also an Honorary Fellow of the University of Edinburgh; a Chair Professor with Shandong University; a Guest Professor with Huazhong University of Science and Technology, Wuhan, China; an Adjunct Professor with Guilin University of Electronic Technology, Guilin, China; and a Guest Researcher with Xidian University, Xi'an, China. He was a Research Fellow with the University of Agder, Grimstad, Norway, from 2001 to 2005; a Visiting Researcher with Siemens AG-Mobile Phones, Munich, Germany, in 2004; and a Research Assistant with the Technical University of Hamburg-Harburg, Hamburg, Germany, from 2000 to 2001. He has published one book chapter and about 130 papers in refereed journals and conference proceedings. He is leading several projects funded by EPSRC, Mobile VCE, and industry, including the RCUK-funded "U.K.-China Science Bridges: R\&D on (B)4G Wireless Mobile Communications". His current research interests include wireless channel modeling and simulation, cognitive radio networks, vehicular communication networks, green radio communications, cooperative (relay) communications, cross-layer design of wireless networks, multiple-input-multiple-output, orthogonal frequency division multiplexing, ultrawideband, and B4G wireless communications.

Dr. Wang is Fellow of the Higher Education Academy and a member of the Institution of Engineering and Technology and the Engineering and Physical Sciences Research Council Peer Review College. He is currently serving as an Editor of the Wireless Communications and Mobile Computing Journal (Wiley), the Security and Communication Networks Journal (Wiley), and the Journal of Computer Systems, Networks, and Communications (Hindawi). $\mathrm{He}$ also served as an Editor of the IEEE TRANSACTIONS ON WIRELESS Communications from 2007 to 2009. He is the leading Guest Editor of the IEEE Journal on Selected Areas in Communications Special Issue on "Vehicular Communications and Networks." He served or is serving as a Technical Program Committee (TPC) Member, TPC Chair, and General Chair for about 60 international conferences. He is listed in the Dictionary of International Biography 2008 and 2009, Who's Who in the World 2008 and 2009, Great Minds of the 21st Century 2009, and 2009 Man of the Year.

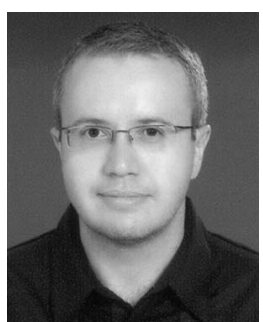

Murat Uysal (SM'07) was born in Istanbul, Turkey, in 1973. He received the B.Sc. and M.Sc. degrees in electronics and communication engineering from Istanbul Technical University in 1995 and 1998, respectively, and the Ph.D. degree in electrical engineering from Texas A\&M University, College Station, in 2001.

Since 2002, he has been with the Department of Electrical and Computer Engineering, University of Waterloo, Waterloo, ON, Canada, where he was promoted to Associate Professor with tenure in 2007. He is currently on leave with Özyeğin University, Istanbul. His specific research areas include multiple-input-multiple-output communication techniques, space-time coding, cooperative diversity, channel estimation/ equalization, multicarrier communication, and wireless optics. His general research interests are in communications theory and signal processing for communications, with particular emphasis on wireless applications.

Dr. Uysal is an Associate Editor of the IEEE TRANSACTIONS ON WIRELESS COMMUNiCATIONS and the IEEE COMmunications LetTers. He was a Guest Coeditor of Wiley's Journal on Wireless Communications and Mobile Computing Special Issue on "MIMO Communications" (October 2004) and the IEEE Journal on Selected AReas in Communication Special Issue on "Optical Wireless Communications" (December 2009). Over the years, he has served on the technical program committees of more than 60 international conferences in the communications area. He cochaired the 2007 IEEE International Conference on Communications (Communication Theory Symposium) and the 2008 IEEE Canadian Conference on Electrical and Computer Engineering (Communications and Networking Symposium). 


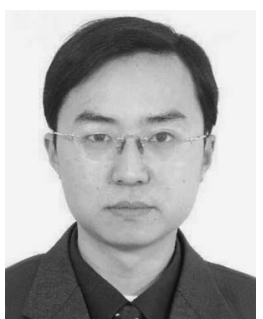

Xiaohu Ge (M'09) received the Ph.D. degree in communication and information engineering from Huazhong University of Science and Technology (HUST), Wuhan, China, in 2003.

He has been with HUST since December 2005, where he is currently an Associate Professor with the Department of Electronics and Information Engineering. Prior to joining HUST, he was an Assistant Researcher with Ajou University, Suwon, Korea, and the Politecnico di Torino, Torino, Italy, from January 2004 to December 2005. He is a project review expert for China's Ministry of Science and Technology and the National Natural Science Foundation of China. His research interests are in the area of mobile communication, traffic modeling in wireless networks, and interference modeling in wireless communications.

Dr. Ge serves as an Editor of the international journal KSII Transactions on Internet and Information Systems. Since 2005, he has been actively involved in the organization of more than 10 international conferences, such as a Publicity Chair for IEEE EuropeComm 2011 and a Cochair of the Green Communication of Cellular Networks Workshop at the 2010 IEEE/ACM International Conference on Green Computing and Communications.

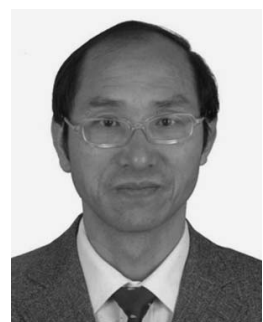

Shan Ouyang received the B.S degree in electronic engineering from Guilin University of Electronic Technology, Guilin, China, in 1986 and the M.S. and $\mathrm{Ph} . \mathrm{D}$. degrees in electronic engineering from Xidian University, Xi' an, China, in 1992 and 2000, respectively.

In 1986, he jointed Guilin University of Electronic Technology, where he is currently a Professor and the Dean of the School of Information and Communications. From June 2001 to May 2002, he was a Research Associate with the Department of Electronic Engineering, The Chinese University of Hong Kong, Shatin, Hong Kong. From January 2003 to January 2004, he was a Research Fellow with the Department of Electronic Engineering, University of California, Riverside. His research interests are mainly in the areas of signal processing for communications and radar, adaptive filtering, and neural network learning theory and applications.

Dr. Ouyang was the recipient of the Outstanding Youth Award of the Ministry of Electronic Industry and Guangxi Province Outstanding Teacher Award, China, in 1995 and 1997, respectively, and the National Excellent Doctoral Dissertation of China in 2002. 